\title{
Constrained two-stream algorithm for calculating aerosol light absorption coefficient from the Particle Soot Absorption Photometer
}

\author{
T. Müller ${ }^{1}$, A. Virkkula ${ }^{2,3,4}$, and J. A. Ogren ${ }^{5}$ \\ ${ }^{1}$ Leibniz Institute for Tropospheric Research, Leipzig, Germany \\ ${ }^{2}$ Finnish Meteorological Institute, Air Quality Research, Helsinki, Finland \\ ${ }^{3}$ University of Helsinki, Department of Physics, Helsinki, Finland \\ ${ }^{4}$ Institute for Climate and Global Change Research, School of Atmospheric Sciences Nanjing, \\ University Nanjing, Nanjing, China \\ ${ }^{5}$ NOAA, Earth System Research Laboratory, Boulder, CO 80305, USA \\ Correspondence to: T. Müller (thomas.mueller@tropos.de)
}

Received: 18 October 2013 - Published in Atmos. Meas. Tech. Discuss.: 18 December 2013

Revised: 8 May 2014 - Accepted: 24 July 2014 - Published: 2 December 2014

\begin{abstract}
We present a new correction scheme for filterbased absorption photometers based on a constrained twostream (CTS) radiative transfer model and experimental calibrations. The two-stream model was initialized using experimentally accessible optical parameters of the filter. Experimental calibrations were taken from the literature and from dedicated experiments for the present manuscript. Uncertainties in the model and calibration experiments are discussed and uncertainties for retrieval of absorption coefficients are derived. For single-scattering albedos lower than 0.8 , the new CTS method and also other correction schemes suffer from the uncertainty in calibration experiments, with an uncertainty of about $20 \%$ in the absorption coefficient. For high single-scattering albedos, the CTS correction significantly reduces errors. At a single-scattering albedo of about 0.98 the error can be reduced to $30 \%$, whereas errors using the Bond correction (Bond et al., 1999) are up to $100 \%$. The correction scheme was tested using data from an independent experiment. The tests confirm the modeled performance of the correction scheme when comparing the CTS method to other established correction methods.
\end{abstract}

\section{Introduction}

Absorption of solar radiation by particles plays an important role in the Earth's radiative balance. The sign of the forcing, which determines whether the particles cause a warm- ing or a cooling of the Earth, strongly depends on the particles' ability to absorb and scatter radiation. Absorption coefficients can vary by many orders of magnitude. For instance, Delene and Ogren (2002) reported yearly averaged values of $0.38 \mathrm{Mm}^{-1}$ for the background station Barrow (Alaska), and Andreae et al. (2008) gave a monthly average absorption coefficient of $91 \mathrm{Mm}^{-1}$ in Guangzhou, a strongly polluted area in China. Generally, the ratios of particle absorption $\left(\sigma_{\mathrm{ap}}\right)$ and scattering coefficients $\left(\sigma_{\mathrm{sp}}\right)$ change depending on the particle composition. The single-scattering albe$\operatorname{dos} \omega_{0}=\sigma_{\mathrm{sp}} /\left(\sigma_{\mathrm{sp}}+\sigma_{\mathrm{ap}}\right)$ were 0.965 and 0.83 for the clean (Barrow) and the polluted (Guangzhou) cases, respectively. Thus it is essential to have methods for measuring the absorption coefficient in environments with very different optical properties and with wide ranges of values.

A recently published review article (Moosmüller et al., 2009) gives an overview of methods for measuring aerosol light absorption. Pros and cons for two categories of methods, filter-based and in situ, are discussed. In this context, in situ denotes methods where the analysis is made while the particles are suspended in air, e.g., photoacoustic photometers, extinction-minus-scattering measurements, and cavity ring-down techniques. In contrast, all filter-based instruments measure particle-related optical parameters after the particles have been deposited on a filter. In situ methods have the advantage of avoiding the contact of particles with the surfaces of the fibers of a filter that might change the absorbing and scattering properties of the particles. Filter-based 
methods measure transmittance or reflectance, or even both, of the particle-filter system. A few methods use the transmittance as a measure for the particle absorption. These methods suffer from a cross sensitivity to particle scattering (Bond et al., 1999; Weingartner et al., 2003; Virkkula et al., 2005; Arnott et al., 2005; Müller et al., 2011), as explained later in this section, but the instruments are generally less expensive and easier to operate than in situ instruments. Consequently, all of the long-term data sets of aerosol light absorption to date have been obtained with filter-based instruments.

The most widely used fiber-filter-based absorption measurement techniques are developed from the integrating plate method (IPM), although the IPM introduced by Lin et al. (1973) does not make use of fiber filters but instead uses polycarbonate membrane filters. The measurement principle to relate the transmittance to the particle absorption and not to the particle extinction was also used for filter-based absorption photometers, since fiber filters act as angular integrating media through multiple scattering of light inside the filter. There are several instruments based on this idea, e.g., the Aethalometer (Hansen et al., 1984), the Particle Soot Absorption Photometer (PSAP; Radiance Research, Seattle, WA), and the Continuous Soot Monitoring System (COSMOS; Miyazaki et al., 2008). Since particles are embedded in a multiple-scattering medium, corrections have to be developed to derive the particle absorption from measurements of transmittance. Another instrument, the Multi-Angle Absorption Photometer (MAAP; Petzold and Schönlinner, 2004), also measures reflection to correct for a further artifact of particle scattering.

Section 2 gives an overview of often-used measurement systems and correction methods. The two-stream radiative transfer model for relating the particle absorption to the transmittance of the filter is introduced in Sect. 3. Calibration experiments for deriving model parameters are given in Sect. 4. An error analysis of the new correction scheme and comparison to existing correction methods are given in Sects. 5 and 6, respectively.

\section{Measurement principle of filter-based absorption photometers}

Filter-based absorption photometers measure the relative transmittance, which is the decrease in the light transmittance, while sample air is drawn through a filter and particles are deposited on the filter. A schematic of this technique is shown in Fig. 1. Inside a system of filter and deposited particles multiple scattering of light occurs. Nevertheless, the filter attenuation coefficient is calculated using an equation of the form of the Beer-Lambert law, which is not strictly valid if multiple scattering is non-negligible. The filter attenuation coefficient $\sigma$ is calculated by

$\sigma(t)=\frac{A}{Q \Delta t} \ln \left(\frac{I(t-\Delta t)}{I(t)}\right)$

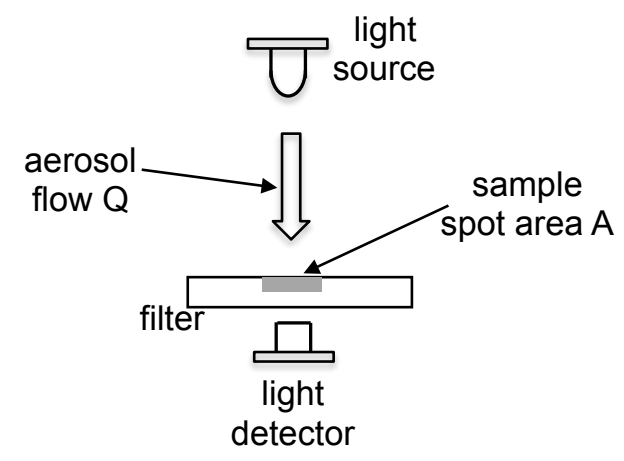

Figure 1. Functional principle of filter-based absorption photometers.

where $A$ is the sample spot area, $Q$ is the sample flow rate, and $I$ is the intensity measured at the beginning and end of the time interval $\Delta t$. An overview of used terms and abbreviations is given in Appendix $C$.

A peculiarity of this method is that more light is absorbed by the deposited particles compared to particles suspended in air. This can be an advantage because the sensitivity to absorption is increased but a disadvantage because the enhancement factor is not constant. This enhanced absorption is attributed to multiple scattering of light by the filter matrix. Insights into the radiative transfer of aerosol-loaded filters have been provided by Clarke et al. (1982), Gorbunov et al. (2002), and Arnott et al. (2005). In laboratory studies it was found that the enhancement factor changes depending on the amount of collected particles. Generally, the enhancement factor becomes smaller with decreasing relative transmittance while loading the filter. Corrections to account for this effect are given for the PSAP in Bond et al. (1999) and Virkkula et al. (2005), and for the Aethalometer in Weingartner et al. (2003) and Arnott et al. (2005). Additionally, it was found that non-absorbing particles reduce the light transmittance, which can be misleadingly interpreted as absorption. This cross sensitivity to particle scattering is called apparent absorption. Corrections for the apparent absorption were developed for Aethalometers (Weingartner et al., 2003; Arnott et al., 2005; Collaud Coen et al., 2010) and PSAPs (Bond et al., 1999; Virkkula et al., 2005). These corrections require simultaneous additional measurements of light-scattering coefficient by nephelometers.

Another filter-based instrument, the Multi-Angle $\mathrm{Ab}$ sorption Photometer (MAAP, Thermo Electron Corporation), measures the transmittance and the reflectance of the particle-laden filter at two angles, and derives the particle absorption using a radiative transfer model (Petzold et al., 2002, 2005; Petzold and Schönlinner, 2004; Hyvärinen et al., 2013). No additional measurement of aerosol scattering is needed. However, in Petzold et al. (2005) and Müller et al. (2011) a remaining cross sensitivity to scattering (defined as the ratio of apparent absorption and scattering coefficient) in the range of $0-3 \%$ was found. 
In recent years several laboratory studies have been conducted to test available photometer corrections for various aerosols. From Müller et al. (2011) it can be concluded that correction methods to account for the apparent absorption need to be revised. It was shown that the cross sensitivity to particle scattering changes while the filter was loaded with scattering particles, and the cross sensitivity was higher at larger wavelengths. For example, when data from PSAP were corrected using the method given in Bond et al. (1999), the cross sensitivities for wavelengths 467,531 , and $650 \mathrm{~nm}$ were on average $0.3,0.4$, and $0.7 \%$, respectively. The apparent absorption became smaller as the filter loading increased, and at a relative transmittance of $70 \%$ the apparent absorption was about $-0.5 \%$ at wavelength $531 \mathrm{~nm}$, i.e., an overcorrection occurred.

\section{Model for particle-laden filters}

Since both the enhancement of absorption and the apparent absorption are functions of the transmittance, it is unlikely that a simple correction can be found with the relative transmittance being the only parameter for the loading state of particle-laden filters. In order to explain measured transmittances, a model for particle-loaded filters was developed. The model includes a two-stream radiative transfer model and parameterizations for the apparent absorption and the absorption enhancement. Since the two-stream model is constrained by the parameterizations, the model is called constrained two-stream (CTS) model. The CTS model is basically a forward calculation to simulate optical properties of the filter with known particle loading. An inversion algorithm is presented for deriving the particle absorption coefficient from transmittances, wherein the effects of particle loading are considered using the CTS model. The combination of the inversion algorithm and CTS model is called the CTS algorithm.

Parameterizations for apparent absorption and absorption enhancement were derived from calibration experiments. Experiments with non-absorbing particles led to a new parameterization of the apparent absorption. Parameterizations of the enhancement effect for absorbing particles were taken from Bond et al. (1999) and Virkkula et al. (2005). The CTS algorithm was compared with the widely used corrections given in Bond et al. (1999) and Virkkula et al. (2005), which are referred to as B1999 and V2005 corrections throughout the rest of the manuscript. The CTS algorithm was developed for the PSAP. However, one can adopt the correction to other types of filter-based absorption photometers.

In the following sections the development of the CTS algorithm is explained in detail. A schematic diagram summarizing the main steps from model initialization to application of the model for deriving absorption coefficients is shown in Fig. 8. The scheme is helpful when reading the sections on the development of the CTS algorithm.

\subsection{Two-stream radiative transfer model}

The radiative transfer of pristine and particle-loaded filters can be described by two-stream models (Bohren, 1987; Arnott et al., 2005; Moteki et al., 2010). In Arnott et al. (2005) such a model was used to derive absorption coefficients from Aethalometers. That model is based on a two-layer system, a composite layer of homogeneously distributed particles in a filter matrix and a particle-free layer of the filter matrix. In Moteki et al. (2010) a two-stream model for a system of many layers is given. With that model a more realistic particle concentration profile following from sampling theory (Lee and Mukund, 2001) can be incorporated into the radiative transfer calculations. Particle concentration profiles depend on many parameters, e.g., particle size, face velocity (average velocity of aerosol perpendicular to the filter) and efficiency coefficients for different collection mechanisms. In Nakayama et al. (2010) it was shown for the PSAP that the absorption enhancement factor of $0.1 \mu \mathrm{m}$ particles can be higher by about $60 \%$ compared to $0.2 \mu \mathrm{m}$ particles, and $0.2 \mu \mathrm{m}$ particles appear to absorb about $23 \%$ more than $0.3 \mu \mathrm{m}$ particles. Thus it is important to consider size effects for evaluation of calibration experiments or atmospheric measurements. In the last years, a few calibration experiments have been performed (Bond et al., 1999; Sheridan et al., 2005; Müller et al., 2011) without considering the size effect. In Moteki et al. (2010), the effect of different particle penetration depths was investigated experimentally. Unfortunately, the reference absorption was modeled by Mie calculations and not measured by an instrument, e.g., a photoacoustic photometer. Since no experimentally verified data on particle penetration depths and related enhancement factors are available, sampling artifacts due to particle sizes are not considered in this manuscript and the simpler two-layer model is used.

Two-stream models are approximations to estimate the intensities transmitted through $\left(I_{\mathrm{t}}\right)$ and reflected from $\left(I_{\mathrm{r}}\right)$ a one-dimensional homogeneous layer when illuminated with an incident intensity $I_{i}$. Here we distinguish between different optical depths. The total optical depth is defined by

$\delta_{\mathrm{tot}}=-\ln \left(\frac{I_{\mathrm{t}}}{I_{\mathrm{i}}}\right)$.

Inside the filter, light is scattered and absorbed by deposited particles or fibers of the filter. We define the extinction optical depth $\delta_{\mathrm{e}}$ as the sum of scattering and absorption optical depths (subscripts s and a) caused by the particle-free filter and airborne particles (subscript $\mathrm{f}$ and $\mathrm{p}$ ). If the particles are embedded in the filter, the relation between extinction and total optical depth is

$\delta_{\mathrm{e}}=\delta_{\mathrm{sf}}+\delta_{\mathrm{sp}}+\delta_{\mathrm{af}}+\delta_{\mathrm{ap}} \neq \delta_{\text {tot }}$.

This means that particle scattering and absorption optical depths in a multiple-scattering environment differ from the 
optical depths for the same particle population in an airborne state because of interference effects. First, there is an interference because the particles are deposited on fibers and do not scatter light independently. Second, the particles are deposited in a multiple-scattering environment because of the high number of light-scnattering fibers in the vicinity of the individual particles. Then the path length of photons passing through the filter becomes larger and the probability of being absorbed increases. Furthermore the particle concentration in the filter increases with time and particles may interfere among each other. There is no theoretical solution describing such interference interactions that we can include in the radiative transfer model. Fortunately, this model uncertainty is implicitly compensated by the CTS model. The calibration experiments are subject to these interferences, which means that the parameterizations of the apparent absorption and absorption enhancement implicitly contain the interference effects. The CTS correction thereby inherits a compensation for the interference effects.

Scattering conserves energy but changes the direction of the propagation of light. The particle asymmetry parameter $g_{\mathrm{p}}$ provides information on the direction in which light is scattered. The probabilities that light is scattered in the forward and backward directions are $\left(1+g_{\mathrm{p}}\right) / 2$ and $\left(1-g_{\mathrm{p}}\right) / 2$, respectively, where $g_{\mathrm{p}}$ is in the range $-1 \leq g_{\mathrm{p}} \leq 1$. In general, asymmetry parameters for particles and fibers $\left(g_{\mathrm{f}}\right)$ differ in their value, and the asymmetry parameter for a system of fibers and particles is calculated by weighting the asymmetry parameters with their corresponding optical depths

$g=\frac{g_{\mathrm{f}} \cdot \delta_{\mathrm{sf}}+g_{\mathrm{p}} \cdot \delta_{\mathrm{sp}}}{\delta_{\mathrm{sf}}+\delta_{\mathrm{sp}}}$.

Therein the particle asymmetry parameter is the average weighted asymmetry parameter of all particles with

$g_{\mathrm{p}}=\frac{\sum_{i} C_{\mathrm{sp}}^{i} g_{\mathrm{p}}^{i}}{\sum_{i} C_{\mathrm{sp}}^{i}}$,

where $i$ denotes the $i$ th particle with scattering cross section $C_{\mathrm{sp}}^{i}$. The scattering coefficient and scattering cross section of an ensemble of particles are related by $\sigma_{\mathrm{sp}}=\sum_{i} C_{\mathrm{sp}}^{i} N^{i}$, where $N^{i}$ is the particle number concentration. Calculation of the asymmetry parameter using Eq. (4) is a simplification. A better representation of the angular dependence of scattered light would require the knowledge of the scattering phase functions of particles, which in most cases is hard to derive. In contrast, the asymmetry parameter can be estimated easily using nephelometers, as will be shown in Sect. 4.2.
In an optically thick layer with multiple scattering the transmittance $T=I_{\mathrm{t}} / I_{\mathrm{i}}$ and reflectance $R=I_{\mathrm{r}} / I_{\mathrm{i}}$ are given by (Arnott et al., 2005; Schuster, 1905; Bohren, 1987)

$$
R=\frac{\omega_{0}(1-g) \sinh \left(K \delta_{\mathrm{e}} / \mu_{1}\right) / K}{\left[2-\omega_{0}(1+g)\right] \sinh \left(K \delta_{\mathrm{e}} / \mu_{1}\right) / K+2 \cosh \left(K \delta_{\mathrm{e}} / \mu_{1}\right)}
$$

and

$$
T=\frac{2}{\left[2-\omega_{0}(1+g)\right] \sinh \left(K \delta_{\mathrm{e}} / \mu_{1}\right) / K+2 \cosh \left(K \delta_{\mathrm{e}} / \mu_{1}\right)},
$$

where

$$
K=\sqrt{\left(1-\omega_{0}\right)\left(1-\omega_{0} g\right)} .
$$

$\mu_{1}$ is typically between unity and $1 / \sqrt{3}$ for diffuse light propagation (Chandrasekhar, 1950; Sagan and Pollak, 1967; Liou, 2002).

The value of $\mu_{1}$ accounts for the elongation of the path length in the medium because of multiple scattering. In Eqs. (6) and (7), one can see that $\mu_{1}$ scales the extinction optical depth. A value near unity is a limiting case in which multiple scattering is negligible and does not contribute to a path length elongation. For this case $\left(\mu_{1} \rightarrow 1\right)$ and negligible backscattering ( $g \rightarrow 1)$, it easily can be derived that the equation for the transmittance simplifies to the Beer-Lambert law with only the absorption optical depth in the exponent $\left(T=e^{-\delta_{a}}\right)$. A value of $\mu_{1}=1 / \sqrt{3}$ was found to be a suitable value for cases with considerable multiple scattering (Sagan and Pollak, 1967; Lyzenga, 1973). This supports the use of a value of $1 / \sqrt{3}$ in this study and in the previous study of Arnott et al. (2005).

The single-scattering albedo $\omega_{0}$ is defined by

$\omega_{0}=\frac{\delta_{\mathrm{sf}}+\delta_{\mathrm{sp}}}{\delta_{\mathrm{sf}}+\delta_{\mathrm{sp}}+\delta_{\mathrm{af}}+\delta_{\mathrm{ap}}}$.

The solution for a two-layer system (subscript $2 \mathrm{~L}$ ) is given in Gorbunov et al. (2002) to be

$R_{2 \mathrm{~L}}=R_{1}+\frac{\left(1-T_{2}\right) \cdot T_{1}^{2}}{1-R_{1} \cdot\left(1-T_{2}\right)}$

and

$T_{2 \mathrm{~L}}=\frac{T_{1} T_{2}}{1-R_{1} \cdot\left(1-T_{2}\right)}$,

where subscripts 1 and 2 refer to the particle-loaded and the particle-free layers, respectively. Equation (10) is not symmetric with respect to the order of the layers, and implies the convention that layer 1 is facing the light source. Equations (10) and (11) are of relevance, since reflectance and transmittance are the only experimentally accessible quantities. 


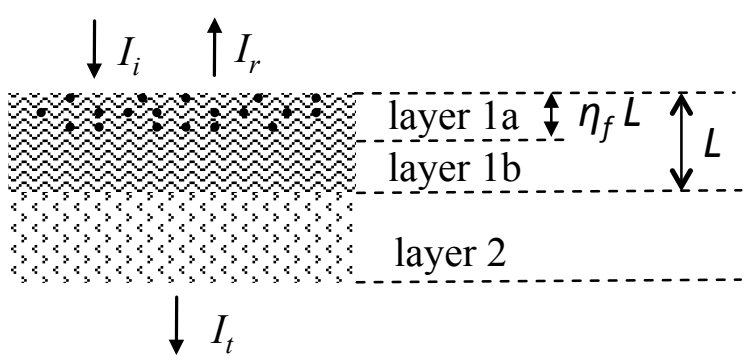

Figure 2. Transmitted and reflected intensities of a filter. The filter consists of two parts: the fiber layer of thickness $\mathrm{L}$ and the backing layer. Particles are collected homogeneously in the upper part of the fiber layer (layer 1a) with the relative thickness $\eta_{\mathrm{f}}$. Layer 2 is the backing layer for the mechanical stability of the filter. In this configuration layer 1 is illuminated directly by the light source. The arrows indicate the incident, reflected, and transmitted radiant flux, respectively.

For non-absorbing layers with $R=1-T$, Eq. (11) becomes symmetric with respect to the order of the layers. In Sect. 3.2 we show that the PSAP filter is only slightly absorbing, and Eq. (11) can be considered to be symmetric. That implies that it does not matter from which side the filter is illuminated. This invariance with respect to the order of layers implies that the filter is illuminated with diffuse light. If filters are illuminated with collimated light, the degree of diffuse or collimated light changes while light travels through the filter. The PSAP was designed to have a mostly diffuse illumination of the sample spot. PSAPs use either opal glass diffusing plates between light source and filter or a diffusely scattering hemisphere for illumination of the spot area. If the filter is illuminated with collimated light, radiative transfer models that account for both diffuse and collimated propagation of light should be used. Such a model is used for the MAAP (Petzold et al., 2005).

Filters used for the PSAP are made up of two different layers. Layer 1 is made of very fine fibers for collecting particles, and layer 2 is a backing layer for mechanical strength. The relative particle penetration depth $\eta_{\mathrm{f}}$ is a dimensionless number between zero and unity and represents the fraction of the layer 1 (cf. Fig. 2) that particles penetrate. An assumption of the model is that particles are deposited homogeneously in that part of the layer facing to the light source. The optical properties of layer 1a are calculated by adding the particle optical depths and the fraction $\eta_{\mathrm{f}}$ of the filter optical depths. Optical properties of layer $1 \mathrm{~b}$ are given by the remaining part (fraction $1-\eta_{\mathrm{f}}$ ) of the filter optical depths.

The relative optical depth of a particle-loaded, two-layer system is defined by

$$
\begin{aligned}
& \delta\left(\delta_{\mathrm{ap}}, \delta_{\mathrm{sp}}, g_{\mathrm{p}}\right)= \\
& -\ln \left(T_{2 \mathrm{~L}}\left(\delta_{\mathrm{ap}}, \delta_{\mathrm{sp}}, g_{\mathrm{p}}, X_{\mathrm{f}}\right)\right)+\ln \left(T_{2 \mathrm{~L}}\left(0,0,0, X_{\mathrm{f}}\right)\right),
\end{aligned}
$$

where $\delta_{\mathrm{sp}}, \delta_{\mathrm{ap}}$, and $g_{\mathrm{p}}$ are particle properties and $X_{\mathrm{f}}=$ $\left\{\delta_{\mathrm{sf}}, \delta_{\mathrm{af}}, g_{\mathrm{f}}, \eta_{\mathrm{f}}, \mu_{1}\right\}$ is a set of parameters defining the optical properties of the pristine filter and the particle concentration profile across the filter. The first term, $-\ln \left(T_{2 \mathrm{~L}},\left(\delta_{\mathrm{ap}}, \delta_{\mathrm{sp}}, g_{\mathrm{p}}, X_{\mathrm{f}}\right)\right)$, is the total optical depth and the second term, $\ln \left(T_{2 \mathrm{~L}},\left(0,0,0, X_{\mathrm{f}}\right)\right)$, is the blank optical depth of an unloaded filter. The relative optical depth is of importance since it is measured by photometers, and the goal is to derive the particle optical depth from the relative optical depth.

The two-stream model is subject to a few assumptions. The parameter $\mu_{1}$ was motivated by the elongation of the light path in a multiple-scattering environment. In our model, $\mu_{1}$ is used for describing the optical properties of the blank filter. Effects of particle loading on $\mu_{1}$ are desirable and would be coupled to the relative penetration depth $\eta_{\mathrm{f}}$. However, loading effects on $\mu_{1}$ can not handled by the model. Furthermore, the assumption of a step function of the particle concentration across the filter is not realistic. From sampling theory the particle profile should be decreasing exponentially in regions of constant particle collection efficiencies. Additionally, the collection efficiency will change with time because of previously collected particles. Other limitations, for example that particles and fibers are not scattering independently, were discussed at the beginning of this section. All these model weaknesses are compensated for by matching the model to experimental calibration functions (cf. Sect. 3.3).

\subsection{Characterization of the pristine filter}

The PSAP uses fiber filters of the type Pallflex E70-2075W (Pall Corp., Ann Arbor, USA). The solution of the radiative transfer problem requires the scattering and absorption optical depths and the asymmetry parameter of the pristine filter, which is a system of two layers. The first layer, layer 1 in our convention, consists of glass fibers and collects almost all particles. The second layer, layer 2, or the backing layer, is important for the mechanical stability but is not effective in terms of particle collection. In Moteki et al. (2010), absorption and scattering cross sections and asymmetry parameters of fibers of the same filter type were calculated by scattering theory using a code for infinite cylinders and oblique incidence of light (Bohren and Huffman, 1983). Fiber diameters were estimated from scanning electron images to be $0.5 \mu \mathrm{m}$ and the complex refractive index of $1.46-0 i$ for fused silica glass was taken from the literature (Smith, 2008). We recalculated the asymmetry parameter using a scattering code for infinite cylinders (http://code.google.com/p/ scatterlib/wiki/Cylinders), which was taken from Bohren and Huffman (1983) and modified by D. Mackowski (personal communication, 2012). The value of 0.84 given in Moteki et al. (2010) could not be reproduced; instead, a value of 0.72 for oblique incidences was derived. There are some issues complicating the exact determination of the filter asymmetry parameter. First, the distribution of fiber diameters may deviate from the diameter of $0.5 \mu \mathrm{m}$; second, the distribution of 


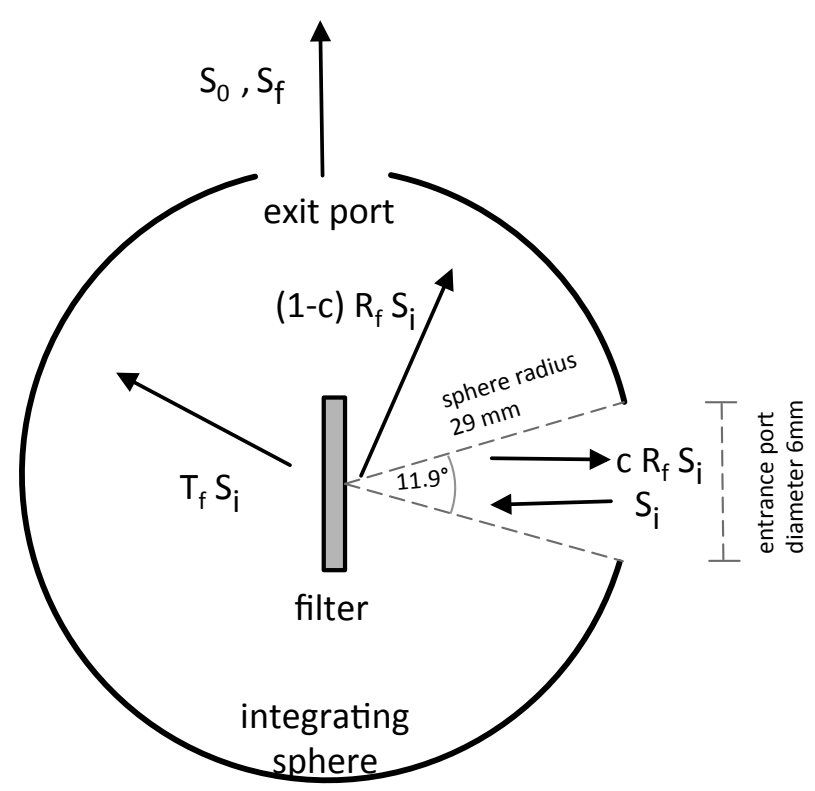

Figure 3. Setup of measurements inside the integrating sphere. The filter is placed in the center of the sphere and illuminated through the entrance port. Reflected and transmitted light is scattered many times on the walls of the sphere before measured at the exit port. The radiant fluxes are indicated by arrows and explained in the text.

angles of incidence is not exactly known; and third, the fibers are touching each other, causing interference effects. For a diffuse illumination we derived an angular-averaged asymmetry parameter, which is about 0.70 for a range of fiber diameters between 0.4 and $0.7 \mu \mathrm{m}$. Average asymmetry parameters for fiber diameters between 0.4 and $3 \mu \mathrm{m}$ would be about 0.65 . To cover the range of values given in Moteki et al. (2010) and this study, we have chosen an asymmetry parameter of 0.75 with an uncertainty of $10 \%$. The sensitivity to the asymmetry parameter and its uncertainty is discussed in Sect. 5.

The filter scattering and absorption optical depths were calculated from measurements of the absorbance using an integrating sphere and from transmittance and reflectance measured with a polar photometer. Measurements were done for both the total filter and for the backing layer. For the latter, the backing layer (layer 2) was isolated by removing the fluffy fiber layer (layer 1). The filter was fixed in the center of an integrating sphere (see Fig. 3). The orientation of the filter was the same as for the PSAP. The filter was illuminated with a spectrally broad UV-VIS light source via an open input port of the sphere. A spectral photometer at the output port of the sphere measured the spectral intensity $S_{\mathrm{f}} . S_{\mathrm{f}}$ can be calculated by a rate equation as

$S_{\mathrm{f}}=\alpha_{\text {sphere }} \cdot T_{\mathrm{f}} \cdot S_{\mathrm{i}}+\alpha_{\text {sphere }} \cdot R_{\mathrm{f}} \cdot(1-c) \cdot S_{\mathrm{i}}$,

where $T_{\mathrm{f}}$ and $R_{\mathrm{f}}$ are the fractions of light transmitted and reflected by the filter, respectively. $S_{\mathrm{i}}$ is the incident intensity and $c$ is the fraction of light that is reflected from the filter and leaves the sphere through the input port. $\alpha_{\text {sphere }}$ is the transmission efficiency of the sphere, which greatly depends on the value of the wall reflectivity. Without a filter inside the sphere, the intensity at the output port is given by

$S_{0}=\alpha_{\text {sphere }} \cdot S_{\mathrm{i}}$.

The value of $c$, which is the relative loss of energy through the input port, can be calculated by assuming a diffuse Lambertian reflectance of the incident light at the surface of the filter. For the sphere with radius $29 \mathrm{~mm}$ and a diameter of the entrance port of $6 \mathrm{~mm}$, light scattered back within a cone with an opening angle of $11.9^{\circ}$ leaves the sphere. This is only valid for an illuminated filter area that is small compared to the diameter of the input port. The $c$ value is the ratio of the reflected energy within the cone and the energy scattered in the backward hemisphere, and is given by

$$
c=\frac{\int_{0}^{11.9^{\circ} / 2} \cos (\theta) \sin (\theta) \mathrm{d} \theta}{\int_{0}^{90} \cos (\theta) \sin (\theta) \mathrm{d} \theta}=0.04
$$

wherein the term $\cos (\theta)$ accounts for the diffuse Lambertian back scattering. The transmission efficiency of the sphere is eliminated by calculating the ratio of intensities measured with and without the filter:

$r_{\mathrm{f}, 0}:=\frac{S_{\mathrm{f}}}{S_{0}}=T_{\mathrm{f}}+(1-c) \cdot R_{\mathrm{f}}$.

The angular intensity $S(\theta)$ was measured with a polar photometer (Fig. 4) in forward $\left(0^{\circ}<\theta<90^{\circ}\right)$ and backward $\left(90^{\circ}<\theta<140^{\circ}\right)$ directions. The angular scattering functions for forward and backward scattering are close to cosine functions, indicating that the light is diffusely transmitted and reflected. Thus, the angular resolution does not contain any information and the reflected intensity was extrapolated to an angle of $\theta=180^{\circ}$ using a cosine dependence. The only useful information from this measurement is the ratio of intensities measured for two angles in transmission and reflection:

$r_{R, T}=S\left(0^{\circ}\right) / S\left(180^{\circ}\right)$.

Measurements of $r_{\mathrm{f}, 0}$ and $r_{R, T}$ were done for the total filter and the backing layer. Results are shown in Fig. 5a. $r_{\mathrm{f}, 0}$ and $r_{R, T}$ were simulated using the two-layer model for the total filter and a single-layer model for the backing layer, respectively (cf. Eqs. 6, 7, 10,11) using the previously estimated asymmetry parameter of 0.75 . Scattering and absorption optical depths for both the filter and the backing layer were found by minimizing the differences between modeled and measured values for $r_{\mathrm{f}, 0}$ and $r_{R, T}$ with an iterative algorithm. It was found by varying the starting values for the scattering optical depths between 0 and 100 and absorption 

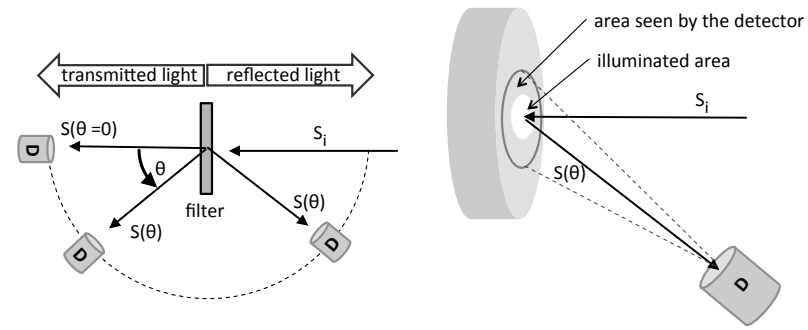

Figure 4. Polar photometer. Left: the filter is illuminated with intensity $S_{\mathrm{i}}$. The detector D can be turned around the center of the filter and measures the intensity of the transmitted and reflected light $S(\theta)$, respectively. Right: the field of view of the detector is sufficiently large that the illuminated area is within the field of view of the detector for all scattering angles $\theta$.

optical depths between 0 and 5 that the solutions for absorption and scattering optical depths are unique. This test was successful for all wavelengths. The resulting optical depths and single-scattering albedos for both layers are shown in Fig. 5b. The single-scattering albedo for the backing layer is slightly lower than the single-scattering albedo for the fiber layer, which is almost unity for a large range of wavelengths. This can also be seen when looking at a filter, since the fiber layer is white and the backing layer is slightly grey.

The scattering optical depths of the fiber layer at the PSAP wavelengths of 467,530 , and $660 \mathrm{~nm}$ are $7.76,7.69$, and 7.34, respectively. The absorption optical depths for these wavelengths are $0.033,0.038$, and 0.018 , respectively. While the values for scattering differ by only $\pm 3 \%$, the absorption shows a higher variation of about $35 \%$. In Sect. 5 we will show that the wavelength dependence of the filter optical depths is negligible. For further investigations we use the optical parameters at the wavelength $530 \mathrm{~nm}$. For comparison, filter parameters from this study and from Moteki et al. (2010) are given in Table 1. The transmittance calculated from Moteki et al. (2010) is an order of magnitude smaller than observations for this study. One reason might be the different asymmetry parameters and the choice of the value for $\mu_{1}$. Since for this study more experimental observations were used for deriving optical filter parameters, the values given in Moteki et al. (2010) are not considered for further investigations.

Uncertainties in the scattering and absorption optical depth were derived from the reproducibility of the experimental results with a set of filters. Systematic errors were estimated to be smaller than $5 \%$. In total, the uncertainties in both the scattering and absorption filter optical depths are about $10 \%$.

The relative particle penetration depth is a simplified model of the true sampling mechanism. According to Moteki et al. (2010), the penetration depth can greatly influence the sensitivity to absorbing particles. We have chosen a relative penetration depth of $\eta_{\mathrm{f}}=0.2$. With this value the calculated enhancement factors are in good agreement with the a) measurements
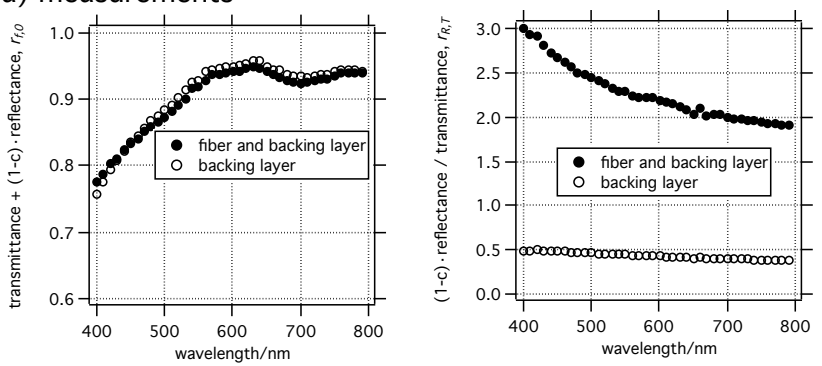

b) modelled optical depths
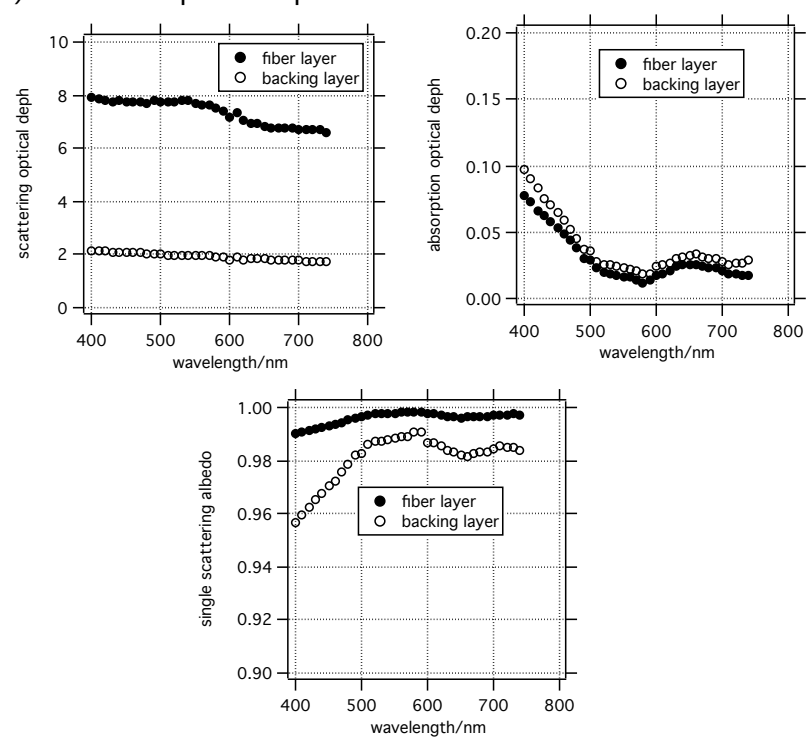

Figure 5. (a) Measured sums and ratios of reflectance and transmittance for the total filter and the backing layer. (b) Modeled scattering and absorption optical depths, as well as single-scattering albedos, for the fiber and backing layers.

enhancement factor for the PSAP given in Bond et al. (1999). Since the effective penetration depth was never measured, we assume an uncertainty of $50 \%$ for the relative penetration depth. Unfortunately, more accurate investigations are almost impossible or at least far out of the scope of this investigation. However, it is important to have a rough idea of the magnitude of the uncertainties. The influence of the uncertainties in filter parameters on the uncertainty in the particle absorption coefficient will be discussed in Sect. 5 .

The relative optical depth of the two-layer system (layers 1a and $1 b$ ) is simulated using Eq. (12) for a range of particle scattering $\left(0<\delta_{\mathrm{sp}}<10\right)$ and absorption $\left(0<\delta_{\mathrm{ap}}<1\right)$ optical depths. Asymmetry parameters were chosen between 0.5 and 0.7 , which is approximately the range of values found in an aerosol monitoring network (Fiebig and Ogren, 2006) for $\mathrm{PM}_{10}$ particles under dry conditions. In Fig. 6a the dependence on particle loading in terms of relative optical depths is shown for a particle asymmetry parameter of $g_{p}=0.6$. The response to purely absorbing particles is independent of the asymmetry parameter and is shown in Fig. 6b. Figure 6c 
A)

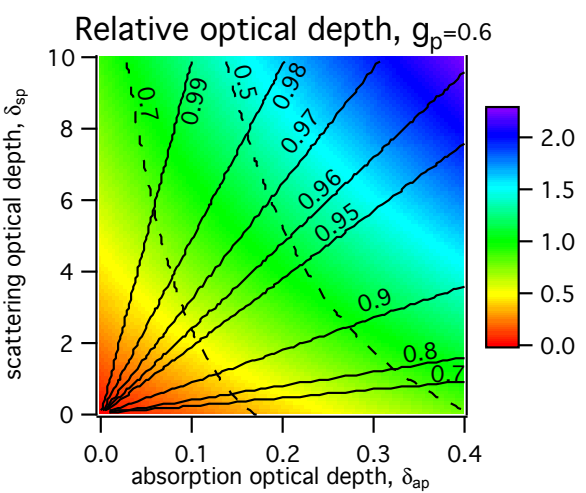

B)

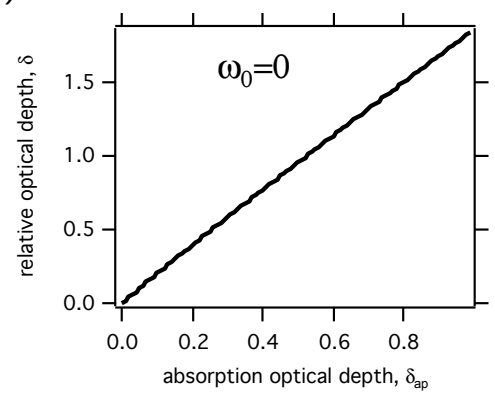

C)

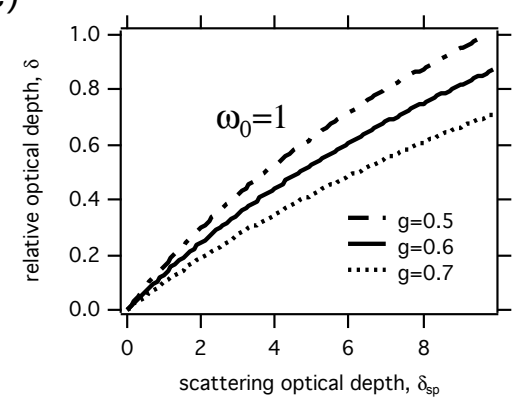

Figure 6. Modeled relative optical depth. (a) Relative optical depth for ranges of particle absorption and scattering optical depths for a particle asymmetry parameter of $g_{\mathrm{p}}=0.6$. The solid lines are isolines for single-scattering albedos and the dashed lines indicate isolines for relative transmittances. (b) Relative optical depth for hypothetical absorbing but not scattering particles. (c) Relative optical depth for loading with non-absorbing particles with particle asymmetry parameters from 0.5 to 0.7 .

shows the dependence of relative optical depth to scatteringonly particles for different values of the asymmetry parameter.

\subsection{Model constraints}

This chapter introduces the concept of the CTS correction scheme.

A two-layer model can be used for modeling the optical properties of particle-loaded filters qualitatively, but not quantitatively. The basic concept for using the model quantitatively is to constrain modeled results to agree with experimental results. The relative optical depth $\delta$ is related to the transmittance when loading a filter with particles (cf. Eq. 12). We define sensitivity functions by

$F_{\mathrm{a}}\left(\delta_{\mathrm{ap}}\right)=\frac{\delta\left(\delta_{\mathrm{ap}}, \delta_{\mathrm{sp}}=0\right)}{\delta_{\mathrm{ap}}}$

and

$F_{\mathrm{s}}\left(\delta_{\mathrm{sp}}, g_{\mathrm{p}}\right)=\frac{\delta\left(\delta_{\mathrm{ap}}=0, \delta_{\mathrm{sp}}, g_{\mathrm{p}}\right)}{\delta_{\mathrm{sp}}}$.

Equations (18) and (19) are the sensitivities to pure black and white particles, respectively. For Eq. (18) the asymmetry parameter is not defined since there is no scattering. In reality, scattering always occurs, so the definition of $F_{\mathrm{a}}$ is merely an idealization of the model. The error introduced by this approximation is smaller than $1 \%$ for filter transmittances between 1.0 and 0.7 . For a mixed population of absorbing and scattering particles, we introduce the mixed-term function $F_{\mathrm{f}}\left(\delta_{\mathrm{ap}}, \delta_{\mathrm{sp}}, g_{\mathrm{p}}\right)$. This function is the ratio of the sum of relative optical depths of pure scattering and absorbing particles to the relative optical depth for the mixed aerosol.

$F_{\mathrm{f}}\left(\delta_{\mathrm{ap}}, \delta_{\mathrm{sp}}, g_{\mathrm{p}}\right)=$
$\frac{\delta\left(\delta_{\mathrm{ap}}=0, \delta_{\mathrm{sp}}, g_{\mathrm{p}}\right)+\delta\left(\delta_{\mathrm{ap}}, \delta_{\mathrm{sp}}=0\right)}{\delta\left(\delta_{\mathrm{ap}}, \delta_{\mathrm{sp}}, g_{\mathrm{p}}\right)}$.

Then the relative optical depth can be written as

$\delta\left(\delta_{\mathrm{ap}}, \delta_{\mathrm{sp}}, g_{\mathrm{p}}\right)=\frac{F_{\mathrm{s}}\left(\delta_{\mathrm{sp}}, g_{\mathrm{p}}\right) \cdot \delta_{\mathrm{sp}}+F_{\mathrm{a}}\left(\delta_{\mathrm{ap}}\right) \cdot \delta_{\mathrm{ap}}}{F_{\mathrm{f}}\left(\delta_{\mathrm{ap}}, \delta_{\mathrm{sp}}, g_{\mathrm{p}}\right)}$.

A fundamental property of $F_{\mathrm{f}}\left(\delta_{\mathrm{ap}}, \delta_{\mathrm{sp}}, g_{\mathrm{p}}\right)$ is that, by definition, it equals unity for non-absorbing $\left(\delta_{\mathrm{ap}}=0\right)$ and nonscattering $\left(\delta_{\mathrm{sp}}=0\right)$ particles. Figure 7 shows $F_{\mathrm{f}}$ for an asymmetry parameter of 0.6 . Single-scattering albedos from 0.7 to 0.99 and relative transmittances of 0.5 and 0.7 are indicated by isolines.

The simulation provides a qualitative insight into the radiative transfer of a system of fibers and particles. However, a model-based correction is limited to assumptions made for 
Table 1. Overview of parameters of fibrous filters. For comparison, values are given for wavelength $550 \mathrm{~nm}$ and for the wavelengths of PSAP.

\begin{tabular}{|c|c|c|c|c|}
\hline \multirow[b]{3}{*}{ Parameter } & \multirow[b]{3}{*}{$\begin{array}{l}\text { Wavel. } \\
\text { [nm] }\end{array}$} & \multicolumn{2}{|c|}{ This study } & \multirow{2}{*}{$\begin{array}{l}\begin{array}{l}\text { Moteki et } \\
\text { al. (2010) }\end{array} \\
\text { Scattering } \\
\text { calculations }\end{array}$} \\
\hline & & \multicolumn{2}{|c|}{$\begin{array}{c}\text { Scattering } \\
\text { calculations } \\
\text { and measurements }\end{array}$} & \\
\hline & & $\begin{array}{l}\text { fiber } \\
\text { layers } \\
\text { 1a and } \\
1 \mathrm{~b}\end{array}$ & $\begin{array}{l}\text { total filter } \\
1 \mathrm{a}, 1 \mathrm{~b} \text { and } \\
2\end{array}$ & total filter \\
\hline \multirow[t]{4}{*}{$n$} & 550 & 1.460 & - & 1.46 \\
\hline & 476 & 1.464 & - & - \\
\hline & 530 & 1.461 & - & - \\
\hline & 660 & 1.459 & - & - \\
\hline$g_{\mathrm{f}}$ & all & $\begin{array}{l}0.75 \pm \\
0.075\end{array}$ & $\begin{array}{l}0.75 \pm \\
0.075\end{array}$ & 0.84 \\
\hline$\mu_{1}$ & all & $\frac{1}{\sqrt{3}}$ & $\frac{1}{\sqrt{3}}$ & 1 \\
\hline$T$ & 550 & $0.31^{\mathrm{a}}$ & - & $0.026^{\mathrm{b}}$ \\
\hline$T+R$ & 550 & $0.971^{\mathrm{a}}$ & - & $0.755^{\mathrm{b}}$ \\
\hline$\omega_{0}$ & 550 & 0.998 & - & 0.996 \\
\hline \multirow[t]{4}{*}{$\delta_{\mathrm{sf}}$} & 550 & $7.63^{c}$ & $2.05^{\mathrm{c}}$ & 120 \\
\hline & 476 & $7.76^{\mathrm{c}}$ & $2.04^{\mathrm{c}}$ & \\
\hline & 530 & $7.69^{c}$ & $2.05^{\mathrm{c}}$ & \\
\hline & 660 & $7.34^{\mathrm{c}}$ & $1.91^{\mathrm{c}}$ & \\
\hline \multirow[t]{4}{*}{$\delta_{\mathrm{af}}$} & 550 & $0.029^{c}$ & $0.035^{\mathrm{c}}$ & 0.45 \\
\hline & 467 & $0.033^{c}$ & $0.040^{c}$ & \\
\hline & 530 & $0.038^{\mathrm{c}}$ & $0.045^{\mathrm{c}}$ & \\
\hline & 660 & $0.019^{c}$ & $0.026^{\mathrm{c}}$ & \\
\hline$\eta_{\mathrm{f}}$ & $\mathrm{n} / \mathrm{a}$ & $0.2^{\mathrm{d}}$ & - & $\begin{array}{l}\text { size- } \\
\text { dependent } \\
\text { penetration } \\
\text { depth }\end{array}$ \\
\hline
\end{tabular}

a $\overline{\text { Measurement. }}{ }^{\mathrm{b}}$ Calculated with Eqs. (18) and (19) in Moteki et al. (2010). ${ }^{\mathrm{c}} 10 \%$ uncertainty. ${ }^{\mathrm{d}} 50 \%$ uncertainty.

simplification. First, the model assumes that particles are uniformly deposited within the top layer of the filter, whereas in a real filter the deposition profile would decrease exponentially (cf. Moteki et al., 2010). Second, the scattering of light by particles and fibers is assumed to be independent, which means that no interference between particle and fiber scattering occurs. Another model simplification is the assumption of a perfectly diffuse illumination. In addition, there might be more artifacts that are not considered in the model. Based on these considerations, an experimental calibration of the theoretical model is indispensable.

From calibration experiments (superscript exp), one can calculate the sensitivities $F_{\mathrm{s}}^{\exp }\left(\delta_{\mathrm{sp}}, g_{\mathrm{p}}\right)$ and $F_{\mathrm{a}}^{\exp }\left(\delta_{\mathrm{ap}}\right)$. However, it is difficult to gather enough data for an experi-

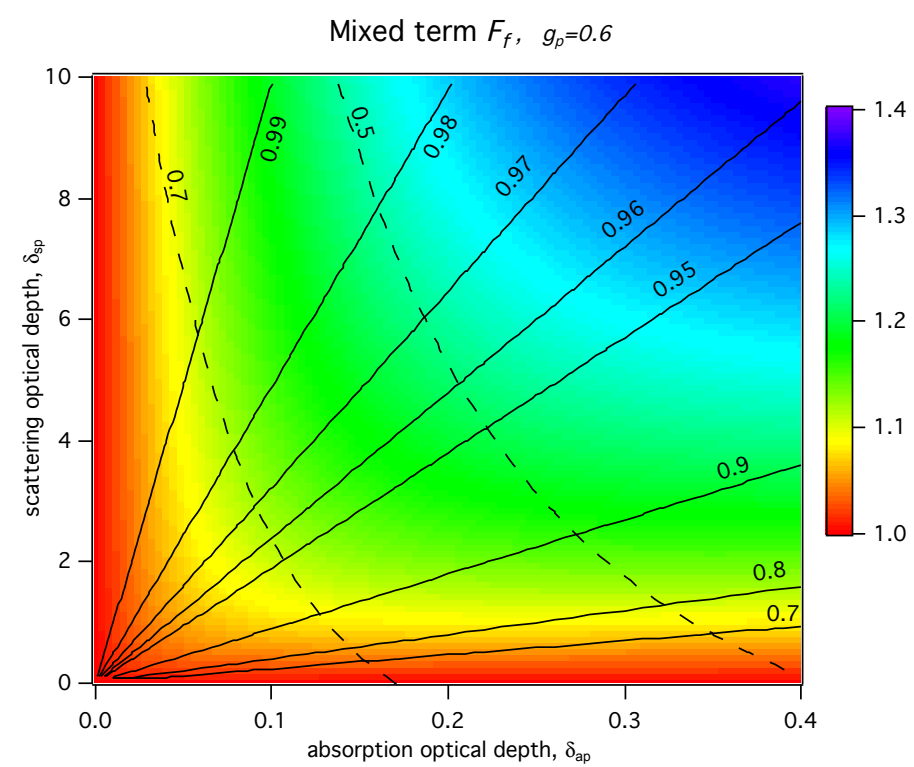

Figure 7. Mixed-term $F_{\mathrm{f}}$ versus scattering and absorption optical depth for a particle asymmetry parameter of 0.6. Shown are lines for single-scattering albedos (solid lines) from 0.7 to 0.99 and for relative transmittances (dashed lines) of 0.5 and 0.7 .

mental determination of the mixed-term $F_{\mathrm{f}}\left(\delta_{\mathrm{ap}}, \delta_{\mathrm{sp}} g_{\mathrm{p}}\right)$, since $F_{\mathrm{f}}$ has three independent variables. Instead, the two-stream model is used for modeling (superscript mod) sensitivities $F_{\mathrm{s}}^{\bmod }\left(\delta_{\mathrm{sp}}, g_{\mathrm{p}}, X_{\mathrm{f}}\right)$ and $F_{\mathrm{a}}^{\bmod }\left(\delta_{\mathrm{a}}, X_{\mathrm{f}}\right)$, where $X_{\mathrm{f}}$ stands for the model parameters of the pristine filter. Then the mixed-term $F_{\mathrm{f}}^{\text {mod }}\left(\delta_{\mathrm{ap}}, \delta_{\mathrm{sp}}, g_{\mathrm{p}}, X_{\mathrm{f}}\right)$ is given by

$F_{\mathrm{f}}^{\bmod }\left(\delta_{\mathrm{ap}}, \delta_{\mathrm{sp}}, g_{\mathrm{p}}, X_{\mathrm{f}}\right)=$

$\frac{F_{\mathrm{s}}^{\mathrm{mod}}\left(\delta_{\mathrm{sp}}, g_{\mathrm{p}}, X_{\mathrm{f}}\right) \cdot \delta_{\mathrm{sp}}+F_{\mathrm{a}}^{\mathrm{mod}}\left(\delta_{\mathrm{ap}}, X_{\mathrm{f}}\right) \cdot \delta_{\mathrm{ap}}}{\delta^{\bmod }\left(\delta_{\mathrm{ap}}, \delta_{\mathrm{sp}}, g_{\mathrm{p}}\right)}$.

The concept for constraining the two-stream model is to combine sensitivity functions for black and white particles derived from experiments and the modeled mixed-term function for grey particles. The relative optical depth then can be written as

$\delta\left(\delta_{\mathrm{ap}}, \delta_{\mathrm{sp}}, g_{\mathrm{p}}\right)=\frac{F_{\mathrm{s}}^{\exp }\left(\delta_{\mathrm{sp}}, g_{\mathrm{p}}\right) \cdot \delta_{\mathrm{sp}}+F_{\mathrm{a}}^{\exp }\left(\delta_{\mathrm{ap}}\right) \cdot \delta_{\mathrm{ap}}}{F_{\mathrm{f}}^{\bmod }\left(\delta_{\mathrm{ap}}, \delta_{\mathrm{sp}}, g_{\mathrm{p}}, \chi_{\mathrm{f}}\right)}$.

The meaning of Eq. (23) is that the model is bound to experimental calibrations for pure black and pure white aerosols, and the mixed-term function can be interpreted as a modeled interpolation for cases when both absorption and scattering occur. The parameters describing the physical properties of the filter are solely used in the modeled mixedterm function. In the following, Eq. (23) is abbreviated as

$\delta=\operatorname{CTS}\left(\delta_{\mathrm{ap}}, \delta_{\mathrm{sp}}, g_{\mathrm{p}} X_{\mathrm{f}}\right)$.

It is assumed that the sensitivity functions from calibration experiments for absorbing and scattering particles implicitly 
include filter sampling artifacts. The radiative transfer model is not able to handle these artifacts, but the CTS algorithm inherently compensates for sampling artifacts from the experimental calibration corrections.

\subsection{Calculation of absorption coefficients}

For deriving the absorption optical depth from Eq. (24), the particle scattering optical depth and the particle asymmetry parameter must be known. In Sect. 4.2 we will show how these parameters are derived from a total and backscattering integrating nephelometer. For calculating the particle absorption optical depth $\delta_{\text {ap }}$, an iterative solver is needed. We used a Newton-type approximation because of its simplicity, but any other solver should work as well. The first step in the iterative solution is to make a first guess of the absorption optical depth $\delta_{\text {ap }}^{0}$ and to calculate the relative optical depth $\delta^{(0)}$ using Eq. (24). Then the absorption optical depth $\delta_{\mathrm{ap}}^{(k)}$ for the $k$ th iteration step is given by

$\delta_{\text {ap }}^{k+1}=$

$\delta_{\mathrm{ap}}^{(k)}-\left(\delta^{(k)}-\delta^{\text {meas }}\right) \cdot\left(\frac{\mathrm{dCTS}\left(\delta_{\mathrm{ap}}^{(k)}, \delta_{\mathrm{sp}}^{\text {meas }}, g_{\mathrm{p}}^{\text {meas }}\right)}{\mathrm{d} \delta_{\mathrm{ap}}^{(k)}}\right)^{-1}$.

The iteration is stopped when the difference between measured and calculated relative optical depth $\left(\delta^{(k)}-\delta^{\text {meas }}\right)$ is small $\left(\sim 10^{-6}\right)$. In most cases the Newton approximation converges with less than 10 iteration steps. The number of iteration steps depends on the filter and scattering optical depths and on the choice of the starting value for $\delta_{\text {ap. }}$ A starting value close to the absorption optical depth of the previous measurement of a time series reduces the number of iterations steps considerably. The CTS function increases strictly monotonically with increasing absorption optical depth. Thus the iterative solver gives a unique solution for the absorption optical depth.

The absorption coefficient is calculated from two consecutive absorption optical depths by

$\sigma_{\text {ap }}(t)=\frac{A}{Q \Delta t}\left(\delta_{\text {ap }}(t)-\delta_{\text {ap }}(t-\Delta t)\right)$,

where $\Delta t$ is the time step between two measurements.

The basic difference between the CTS correction and the B1999 and V2005 corrections is that the CTS correction first corrects the relative optical depth for the scattering and filter artifacts. The result is the absorption optical depth, from which the absorption coefficient is calculated from the change in the optical depth with time. This is a kind of a differentiation but with discrete data. The advantage of first correcting for filter and scattering artifacts is that the loading state is specified by scattering and absorption optical depths. In contrast, the B1999 and V2005 corrections start with a differentiation of the time series of the attenuation. After the differentiation the only loading-related parameter is the relative transmittance, which means that a loss of information has occurred. Afterwards, scattering and filter artifacts are corrected but with less information on the loading state. Additionally the CTS correction accounts for the particle asymmetry parameter, which is not considered in the B1999 and V2005 corrections.

\section{Calibration experiments}

\subsection{Black particles}

The relative transmittance $\tau$ and relative optical depth $\delta$ are derived from the ratio of the actual intensity and the intensity prior to loading the filter, and are defined by (cf. Eq. 2)

$$
\begin{aligned}
& \delta(t)=-\ln (\tau(t))=-\ln \left(\frac{I(t)}{I(0)}\right) \\
& =-\ln \left(\frac{I(t) / I_{\mathrm{i}}}{I(0) / I_{\mathrm{i}}}\right)=\delta_{\mathrm{tot}}(t)-\delta_{\mathrm{tot}}(0) .
\end{aligned}
$$

Equation (27) also shows the connection between relative and total optical depths.

The ratio of particle absorption coefficient and filter attenuation coefficient $(\sigma(t)$ in Eq. 1) is the filter transmission function:

$f_{\mathrm{tr}}\left(\tau(t), \sigma_{\mathrm{sp}}(t)\right)=\frac{\sigma_{\mathrm{ap}}(t)}{\sigma(t)}$.

For black particles the filter transmission function accounts for the enhancement effect due to scattering of light in the fiber matrix. Filter transmission functions for the PSAP were derived during calibration experiments leading to different correction methods, i.e., the B1999 and V2005 corrections. For convenience, the time dependence is omitted throughout the rest of the manuscript.

Calibration of filter-based absorption photometers necessarily requires a reference method for measuring particle absorption. In Bond et al. (1999) the PSAP was calibrated using the difference of extinction minus scattering of airborne particles (unaltered/not collected on filter or other substrate) as the reference. The filter transmission function of the B1999 correction scheme is given by

$f_{\mathrm{tr}, \mathrm{B} 1999}\left(\tau, \sigma_{\mathrm{sp}}, \sigma\right)=\frac{1}{c_{1} \cdot \tau+c_{2}}-s \frac{\sigma_{\mathrm{sp}}}{\sigma}$,

with the constants $c_{1}=1.555, c_{2}=1.023$, and $s=0.0164$. The constants given here differ from values given in Bond et al. (1999), since corrections for spot size and a further wavelength adjustment are included. For details, the reader is referred to Ogren (2010). Scattering coefficients in the B1999 correction are not corrected for the illumination and truncation error (Anderson et al., 1996). For non-scattering particles $\left(\sigma_{\mathrm{sp}}=0\right)$ the filter transmission function is

$f_{\text {tr,B1999, black }}(\tau)=\frac{1}{c_{1} \cdot \tau+c_{2}}$. 


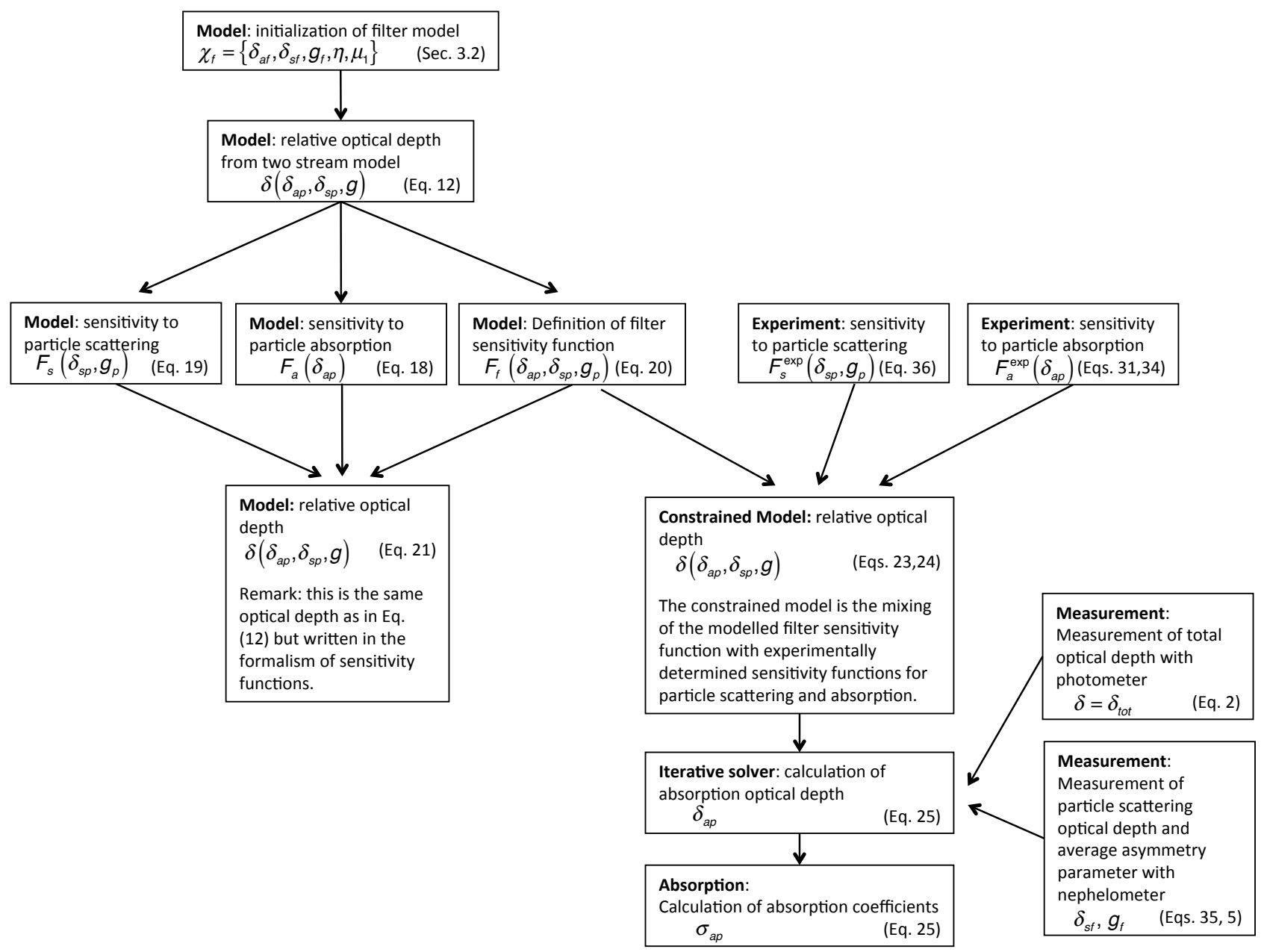

Figure 8. Overview of model implementation, constraints to experimental sensitivity functions, and retrieval of absorption from measurements with absorption photometers and measurements of scattering properties.

Filter transmission functions based on "coefficients" are unhandy since radiative transfer models need optical depths. Equation (30) can be written in terms of relative optical depths by

$\delta\left(\delta_{\mathrm{ap}}\right)=\ln \left(\frac{e^{c_{2} \delta_{\mathrm{ap}}+\ln \left(c_{1}+c_{2}\right)}-c_{1}}{c_{2}}\right)$.

The derivation of Eq. (31) is given in Appendix A.

During the Reno Aerosol Optics Study (RAOS; Sheridan et al., 2005), different reference methods with photoacoustic spectrometers, extinction coefficient minus scattering coefficient, and cavity ring-down instruments were available. Results from the RAOS experiment revealed agreement between photoacoustic and extinction-minus-scattering methods of $\sim 3-7 \%$ for a variety of absorbing aerosols. Experiments with high absorption coefficients up to $800 \mathrm{Mm}^{-1}$ showed differences of less than $3 \%$, indicating the good performance of these two fundamentally different reference methods. Consequently, the average of these two methods was used as the reference absorption for evaluating the performance of filter-based absorption photometers, including MAAPs (Petzold et al., 2005), Aethalometers (Arnott et al., 2005), and PSAPs (Virkkula et al., 2005). In addition to a three-wavelength PSAP with modified optics, a standard single-wavelength PSAP, identical to instruments used in many laboratories and long-term monitoring sites, was compared to the reference absorption. Wavelengths of this type of PSAP are given to be $565 \mathrm{~nm}$ (Radiance Research, Operating manual), $567 \mathrm{~nm}$ (Bond et al., 1999), $574 \mathrm{~nm}$ (Virkkula et al., 2005), and $586 \mathrm{~nm}$ (Müller et al., 2011). The optical wavelength has to be accounted for when comparing to other instruments. For adjusting wavelengths of the PSAP and nephelometer, we refer the reader to Ogren (2010).

In Virkkula et al. (2005) and Virkkula (2010), the filter transmission function is given by

$$
f_{\mathrm{tr}, \mathrm{V} 2005}\left(\tau, \omega_{0}\right)=c_{1}+c_{2}\left(h_{0}+h_{1} \omega_{0}\right) \ln (\tau)-s \frac{\sigma_{\mathrm{sp}}}{\sigma},
$$




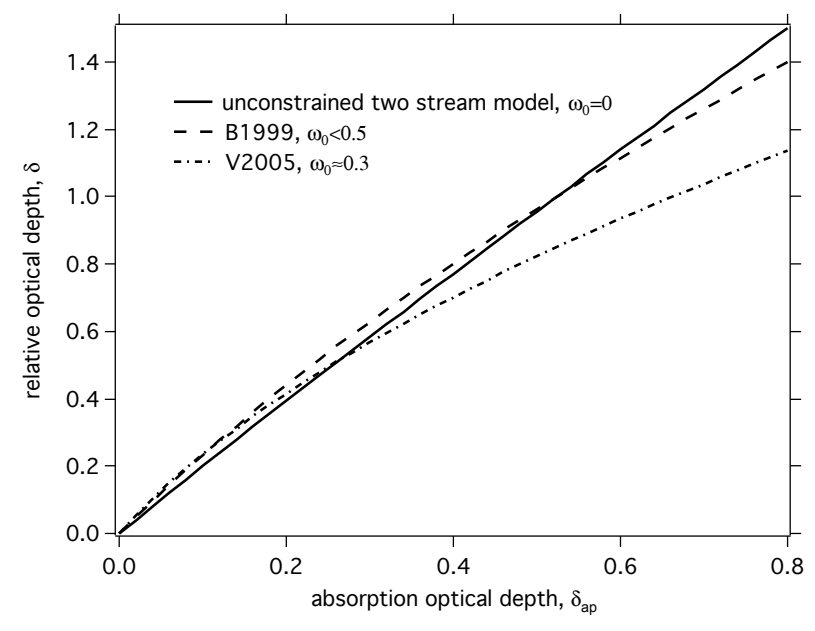

Figure 9. Comparison of the unconstrained two-stream model and experimental calibrations in B1999 and V2005. Shown are the relative optical depths versus the particle absorption optical depth.

with $\quad c_{1}=0.354 \pm 0.009, \quad c_{2}=-0.617 \pm 0.016, \quad h_{0}=$ $1.192 \pm 0.219, h_{1}=-0.800 \pm 0.336$, and $s=0.023$ for the single-wavelength PSAP. Values for a three-wavelength PSAP were not used, since that instrument was a modified prototype that differs slightly from the commercially available multi-wavelength PSAP. Equation (32) can only be solved iteratively, but for black particles with $\omega_{0}=0$ and $\sigma_{\mathrm{sp}}=0$ the analytical solution is

$f_{\mathrm{tr}, \mathrm{V} 2005}(\tau)=c_{1}+c_{2} h_{0} \ln (\tau)$.

Equation (33) expressed as relative optical depth is

$\delta\left(\delta_{\mathrm{ap}}\right)=\sqrt{\left(\frac{c_{1}}{c_{2} h_{0}}\right)^{2}-\frac{2 \delta_{\mathrm{ap}}}{c_{2} h_{0}}}+\frac{c_{1}}{c_{2} h_{0}}$.

The derivation of Eq. (34) is given in Appendix B.

There are obvious differences between the filter transmission functions of the B1999 and V2005 corrections (Fig. 9) that are not yet understood. However, the purpose of this manuscript is not to present a new calibration with black particles but to introduce a new concept based on a radiative transfer model. We will evaluate the sensitivity of the CTS method to these two filter transmission functions.

\subsection{White particles}

The response of PSAPs to white particles was measured in the laboratory. A solution of $\mathrm{NaCl}$ was nebulized, passed through a cyclone, and dried. Effective particle sizes and thus particle asymmetry parameters were varied by changing the operating conditions of the nebulizer. The response to $\mathrm{NaCl}$ particles was measured by two three-wavelength PSAPs and a three-wavelength, total and backscattering, integrating nephelometer (TSI, model 3563). Scattering coefficients were corrected for angular non-idealities using the parameterization given in Anderson and Ogren (1998). The scattering optical depth was calculated for the PSAPs by

$\delta_{\mathrm{sp}, n}=\frac{Q \Delta t}{A} \sum_{i=1}^{n} \sigma_{\mathrm{sp}, i}$.

The summation starts with the beginning of loading the filter. Asymmetry parameters for each wavelength were determined from the measured hemispheric backscattering fraction using a relation given in Andrews et al. (2006). Asymmetry parameters $g_{\mathrm{p}}$ ranged from about 0.45 to 0.68 . Figure 10a shows the measured dependence of the relative optical depth on the scattering optical depth. It can be seen that there is a pronounced dependence on the particle asymmetry parameter. A parameterization describing this dependence was found to be

$\delta\left(\delta_{\mathrm{sp}}, g_{\mathrm{p}}\right)=\delta_{\mathrm{sp}} \cdot\left(a_{5}+\left(a_{0}+a_{1} g_{\mathrm{p}}\right) \cdot e^{-\left(\frac{\ln \left(\delta_{\mathrm{sp}}\right)+a^{2}}{a_{3}+a_{4} g_{\mathrm{p}}}\right)^{4}}\right)$,

with $\quad a_{0}=0.1509, \quad a_{1}=-0.1611, \quad a_{2}=4.5414$, $a_{3}=-5.7062, a_{4}=-1.9031$, and $a_{5}=0.01$. The choice of a suitable parameterization was motivated by (i) having a constant, (ii) a term with linear dependence on the asymmetry parameter, and (iii) a loading term with an exponential decrease. Figure 10b shows relative differences between measured and parameterized relative optical depths.

Equation (36) accounts for two particle size effects. Smaller particles usually have smaller asymmetry parameters. Furthermore, smaller particles penetrate deeper into the filter, which influences the transmittance as discussed in the context of a size-dependent absorption enhancement. Thus, there seems to be a coupling between asymmetry parameter and particle penetration depth. No measurements with a particle size spectrometer were done. Thus we can not separate these two effects from the measurements. Nevertheless, because of the coupling, the effect of a size-dependent particle penetration depth is implicitly considered by the particle asymmetry parameter. A detailed analysis of the strength of the coupling is beyond the scope of this manuscript and requires more experiments.

In Fig. 10a and b, two families of curves can be seen. One family occurred for small particles with scattering Ångström exponents of 2.5 and asymmetry parameters between 0.615 and 0.476 . The other family of curves is for larger particles with scattering Ångström exponents between 2.0 and 1.0 and asymmetry parameters ranging from 0.684 to 0.613 . The relative deviation between fitted and measured curves indicates that the branch with the higher scattering Ångström exponents is not well described by the parameterization since the deviation is up to $20 \%$. A satisfactory explanation for this behavior was not found. Measurement uncertainties can be excluded, since nephelometers typically have errors smaller than $5 \%$, and the deviations between the two PSAPs used for these experiments were smaller than $2 \%$. A possible 

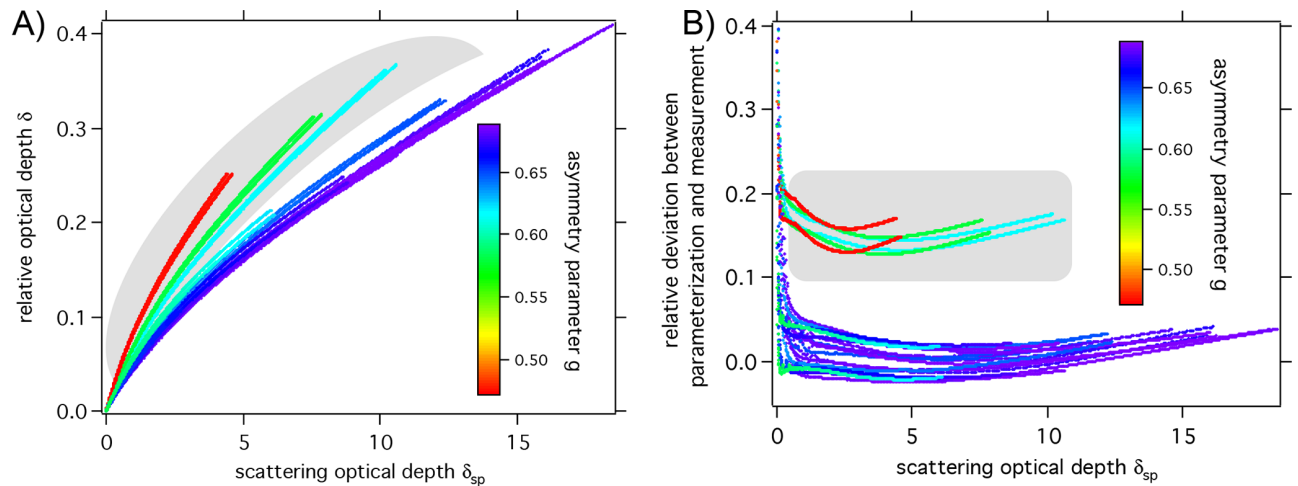

Figure 10. (a) Measured relative optical depths versus measured scattering optical depths for the three wavelengths of two PSAPs. The particle asymmetry parameter is color coded. (b) Relative deviation of measured and parameterized optical depths defined by $\left(\delta^{\text {meas }}-\delta^{\text {param }}\right) / \delta^{\text {meas }}$. The greyish areas indicate data with high scattering Ångström exponents, for which the parameterization has a higher uncertainty.

explanation could be an invalid model assumption that the asymmetry parameter and the scattering optical depth solely describe the scattering artifact. Furthermore the calculation of the asymmetry parameter from the measured backscatter fraction might be inaccurate for particle populations with high scattering Ångström exponents. However, the maximum deviation of about $20 \%$ is much smaller than the $100 \%$ uncertainty in the scattering correction given in Bond et al. (1999). Thus a deeper investigation of this issue was not done.

The error of the scattering parameterization is calculated by the root mean square of the relative deviation between parameterization and measurement:

$\Delta \delta_{\mathrm{rms}}=\sqrt{\frac{1}{N} \sum\left(\frac{\delta^{\text {param }}-\delta^{\text {meas }}}{\delta^{\text {meas }}}\right)^{2}}$.

Mathematically this is identical to the standard deviation of the relative deviation. The error is about $9 \%$ for all data and $7 \%$ when excluding noisy data at low loadings $(\delta<0.2)$ and excluding small asymmetry parameters $(g<0.55)$, which are relatively rare for ambient aerosols (Fiebig and Ogren, 2006) and correspond to the branch with scattering Ångström exponents of 2.5 .

\section{Error analysis}

\subsection{General approach}

The errors of the CTS algorithm due to one or more erroneous input parameters are investigated by means of error propagation. Generally, if $\Psi$ is a function of $i$ independent input parameters $\Phi_{i}, \Psi=F\left(\Phi_{i}\right)$, the error of $\Psi$ is calculated by

$\Delta \Psi=\sqrt{\sum_{i}\left(\frac{\partial F\left(\Phi_{i}\right)}{\partial \Phi_{i}} \Delta \Phi_{i}\right)^{2}}$, where $\Delta \Phi_{\mathrm{i}}$ is the error of $\Phi_{\mathrm{i}}$. The $F$ functions of interest are Eqs. (24), (25), and (26) for the relative optical depth, absorption optical depth, and absorption coefficient, respectively. Although the $F$ functions are results of an iterative solver, the $F$ functions are sufficiently smooth to allow for a numerical calculation of the local derivatives. Errors are calculated for ranges of scattering (0 to 10$)$ and absorption (0 to 1) optical depths to investigate the errors for different possible loading states.

\subsection{Wavelength dependence of filter optical parameters}

In this section we investigate the influences of the wavelength dependencies of the filter scattering and absorption optical depths on the retrieval of the particle absorption optical depth. Equation (25) is used as the $F$ function in Eq. (38). The uncertain variables $\left(\Phi_{\mathrm{i}}\right)$ are the filter absorption and scattering optical depths, with values of $\delta_{\mathrm{sf}}=7.62$ and $\delta_{\mathrm{af}}=0.029$ and a relative variability of $\Delta \delta_{\mathrm{sf}} / \delta_{\mathrm{sf}}=3 \%$ and $\Delta \delta_{\mathrm{af}} / \delta_{\mathrm{af}}=35 \%$ at the wavelengths of a three-wavelength PSAP (cf. Sect. 3.2). The variability of the filter scattering optical depth causes an uncertainty smaller than $0.55 \%$ for single-scattering albedos near unity and becomes even smaller with decreasing single-scattering albedo. At a singlescattering albedo of 0.9 the uncertainty is smaller than $0.17 \%$. Similarly, the uncertainties due to the wavelength dependence of the filter absorption optical depth are 0.47 and $0.23 \%$ at single-scattering albedos of 1 and 0.9 , respectively, and become smaller with decreasing single-scattering albedos. The worst case of the combined errors for both uncertainties is smaller than $1 \%$. These results show that there is no need to consider the wavelength dependence of filter parameters for the three wavelengths of the PSAP. 


\subsection{Prediction of relative optical depth}

In this section we investigate the error for predicting the relative optical depth. Discussion of this error is easy since the iterative solver is not needed. Uncertainties influencing the prediction of the relative optical depth (Eq. 24) are uncertainties in the filter parameters and the uncertainties in the particle optical depths.

The filter parameters (and relative uncertainties) are $\delta_{\mathrm{sf}}=$ $7.62(10 \%), \delta_{\mathrm{af}}=0.029(10 \%), g_{\mathrm{f}}=0.75(10 \%)$, and $\eta_{\mathrm{f}}=$ $0.2(50 \%)$. Relative uncertainties in the relative optical depth from the individual contributions are shown in Fig. 11 for particle scattering optical depths from 0 to 10 and particle absorption optical depths between 0 and 0.5 , with a particle asymmetry parameter of 0.7 . The largest uncertainties emerge due to the uncertainties in the filter asymmetry parameter and particle penetration depth. In general, uncertainties are largest for single-scattering albedos between 0.95 and 0.98 . For single-scattering albedos $\omega_{0}=0$ and $\omega_{0}=1$, the model uncertainties tend towards zero, since for these limiting cases the relative optical depth is completely determined by the experimental calibrations. The approach of constraining the radiative transfer model to experimental calibrations suppresses uncertainties in the input parameters to the model output.

The error in predicted relative optical depths due to uncertainties in the particle optical depths and asymmetry parameters is discussed in the following. Uncertainties in correction schemes for black particles are on the order of $20 \%$ (Bond et al., 1999), including uncertainties in the PSAP and reference instruments. In Virkkula et al. (2005), the uncertainty in the parameterization is given to be about $3 \%$, whereas uncertainties in the reference absorption were not considered. A comparison of B1999 and V2005 corrections given in Baumgardner et al. (2012) affirmed that the error is of the order of $20 \%$. Errors of the scattering optical depth result from calibration uncertainties of about $3 \%$ (Heintzenberg et al., 2006) and from uncertainties in the truncation error. For investigating the scattering artifacts we used the truncation correction given in Anderson and Ogren (1998). With this method the error in the scattering coefficient is about $2 \%$ for a wide range of atmospheric aerosols and can be up to $5 \%$ for strongly absorbing particles (Bond et al., 2009). In the following the total uncertainty in the scattering optical depth is assumed to be $7 \%$. Asymmetry parameters can be estimated from a parameterization of the backscatter fraction measured with nephelometers (Andrews et al., 2006). Fiebig and Ogren (2006) compared asymmetry parameters derived with this method and asymmetry parameters retrieved by an inversion algorithm from data of monitoring stations with aerosols classified as being arctic, continental, and marine. Differences of 3 to $4 \%$ and in extreme cases up to $14 \%$ were attributed to a large part to assumptions made in calculating the asymmetry parameter from the measured backscatter fraction. For further error analysis we used an uncertainty in the asymmetry parameter of $10 \%$, which lies between the expected and extreme values. The error of the relative optical depth is shown in Fig. 12, for $7 \%$ error of the particle scattering (panel a), for $20 \%$ of the particle absorption (panel b), and for $10 \%$ of the particle asymmetry parameter (panel c).

The total error of the relative optical depth including uncertainties in the filter and particle parameters is shown in Fig. 13a. The same data are plotted as a function of the single-scattering albedo in Fig. 13b. There is no large variation of the uncertainty between single-scattering albedos from 0.2 to 0.95 . In this range the error is dominated by the error of the absorption measurement. For single-scattering albedos above 0.95 , the errors of the scattering and asymmetry parameters dominate. Comparing Figs. 11 and 12, it can be seen that the model uncertainties are small compared to uncertainties in the calibration measurements.

\subsection{Error of retrieved absorption coefficients}

The error of the retrieved absorption optical depth was derived similarly. The absorption optical depth is calculated using Eq. (25). The error is calculated considering all uncertainties, including the uncertainties in filter parameters, particle scattering optical depth and asymmetry parameter, and uncertainties in the PSAP calibration. The black particle calibration was taken from the B1999 correction. The relative uncertainty $\Delta \sigma_{\mathrm{ap}} / \sigma_{\mathrm{ap}}$ of this CTS-B1999 correction is shown in Fig. 14a. Similarly the uncertainty was calculated for the original B1999 correction. Relative errors for the CTSB1999 and B1999 correction are shown in Fig. 14b versus the single-scattering albedo. For low single-scattering albedos the uncertainties are similar for both methods, since the parameterization for black particles was taken from the B1999 correction. At high single-scattering albedos the uncertainty in the CTS correction is much smaller than the B1999 correction, e.g., the uncertainty level of $50 \%$ is reached at a singlescattering albedo of 0.99 and 0.95 for the CTS-B1999 and for the B1999 corrections, respectively.

\section{Comparison of correction methods}

In this chapter we show results of a re-evaluation of data from the RAOS experiment (Sheridan et al., 2005), in which absorption coefficients were measured using a PSAP (Virkkula et al., 2005) and scattering and backscattering were measured with a nephelometer. Scattering coefficients were corrected for the truncation error using the method shown in Anderson and Ogren (1998). Furthermore, the scattering coefficients were adjusted to the wavelengths of the PSAP by use of the scattering Ångström exponents. Asymmetry parameters were derived from the backscatter fraction as shown in Andrews et al. (2006). The reference absorption was determined from the average of photoacoustic spectrometers and extinction minus 


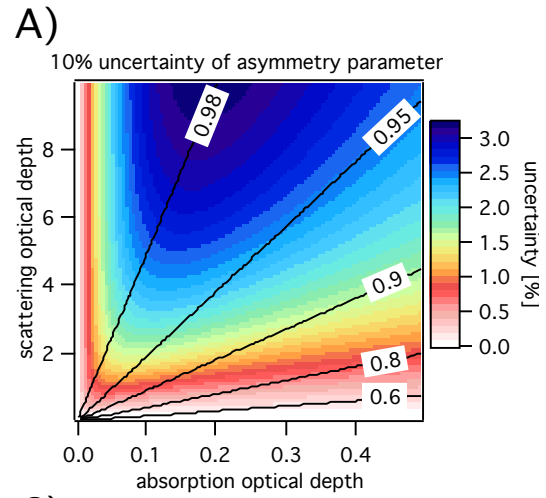

C)

B)
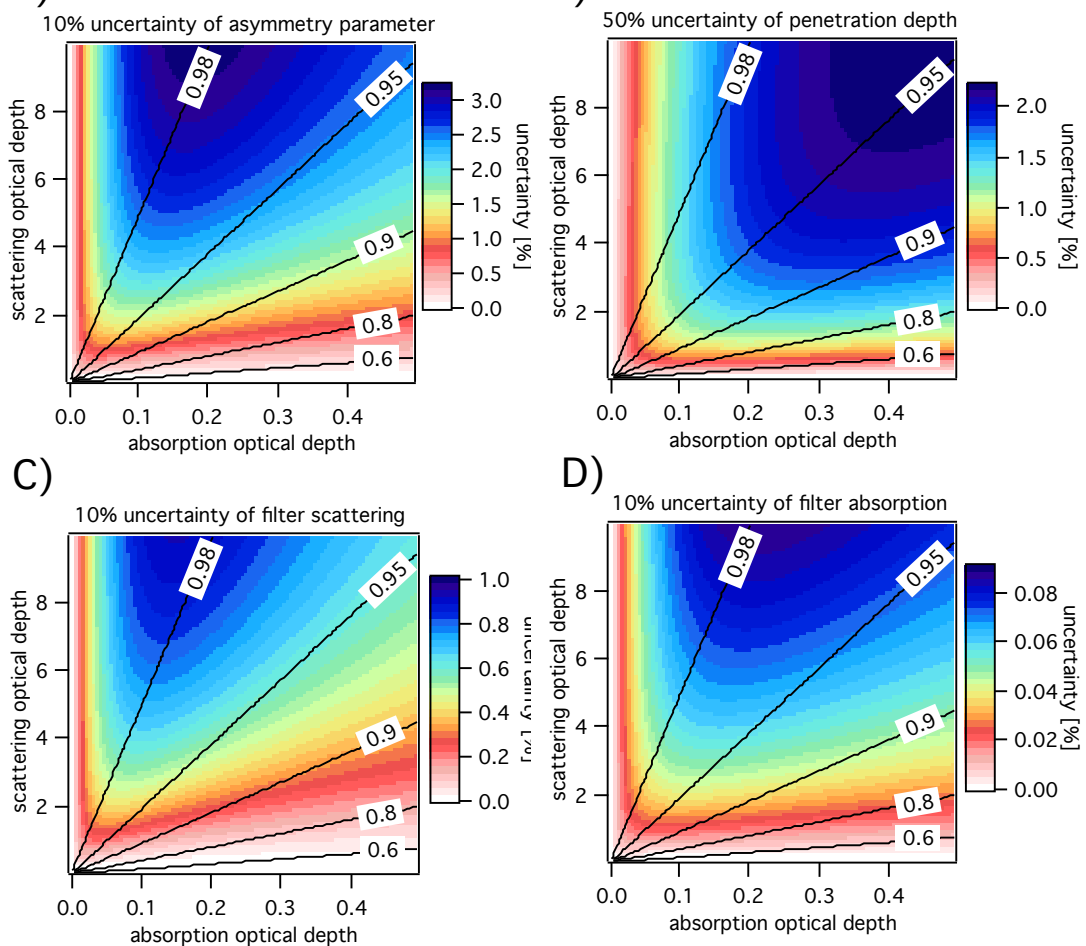

D)

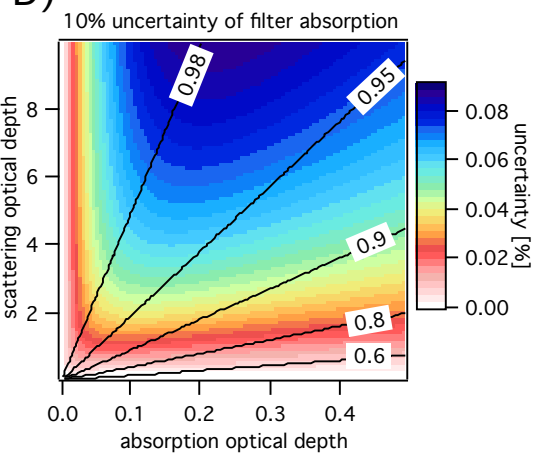

Figure 11. Relative uncertainties in the relative optical depth caused by uncertainties in (a) filter asymmetry parameter; (b) particle layer thickness; and (c) and (d) filter scattering and absorption optical depth, respectively. Uncertainties were calculated for ranges of particle scattering and absorption optical depth and for a particle asymmetry parameter of 0.7 . The solid lines indicate constant single-scattering albedos.

scattering (Sheridan et al., 2005). Values from the PSAP were corrected using the B1999, V2005, and CTS methods.

Data from several experiments were classified according to the single-scattering albedo. The ranges of singlescattering albedos (number of experimental runs, and the total time in minutes) are 0.98 to 0.97 ( 7 runs, $332 \mathrm{~min}$ ), 0.95 to 0.94 ( 2 runs, $129 \mathrm{~min}$ ), 0.9 to 0.86 ( $9,400 \mathrm{~min}), 0.84-0.7$ (9, $415 \mathrm{~min}$ ), black (2, $104 \mathrm{~min})$, and white (13 runs, $755 \mathrm{~min}$ ). Black denotes a range of single-scattering albedos from 0.25 to 0.6 , and white stands for a single-scattering albedo of unity. For the CTS correction, black particle loading corrections from both B1999 (Eq. 31) and V2005 (Eq. 34) were used. The corrections are denoted as CTS-B1999 and CTSV2005, respectively. Ratios of corrected absorption coefficients and the reference absorption were calculated. Figure 15 shows median values and 25 th and 75 th percentiles for all classes of single-scattering albedos. For experiments with white particles, apparent absorption coefficients divided by the scattering coefficient are shown. It can be seen that the ratios for CTS-V2005 and V2005 scheme are close to unity for black particles and single-scattering albedos between 0.7 and 0.84 . This is not surprising since the V2005 correction and accordingly also the black particle correction for CTS-V2005 were derived from the same data set from the
RAOS study. For higher single-scattering albedos between 0.95 and 0.98 , the ratios are 1.52 for B1999; 1.16 for V2005; and 1.07 and 1.04 for CTS-B1999 and CTS-V2005, respectively. For single-scattering albedos above 0.9 , the span between the 25th and 75th percentiles is significantly smaller for the CTS corrections compared to B1999 and V2005. This and the better ratio at high single-scattering albedos can be explained by considering a parameterization for the scattering and asymmetry parameter in the new loading correction. For white particles, the ratios of apparent absorption and scattering for CTS, B1999, and V2005 corrections are $0.0008,0.015$, and 0.005 , respectively. The spans of 25 th and 75th percentiles for CTS are remarkably smaller compared to B1999 and V2005. The method comparison shows that the concept of CTS significantly reduces uncertainties in the particle absorption at high single-scattering albedos.

\section{Summary and outlook}

A new method, the constrained two-stream (CTS) method, for correcting filter-based absorption photometers was developed. The method is basically applicable to any instrument whose measurement principle is based on the measurement of the light attenuation of a particle-loaded fiber filter. For ap- 
A)

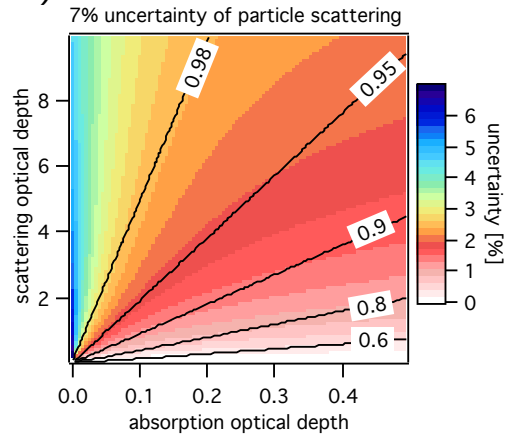

B)

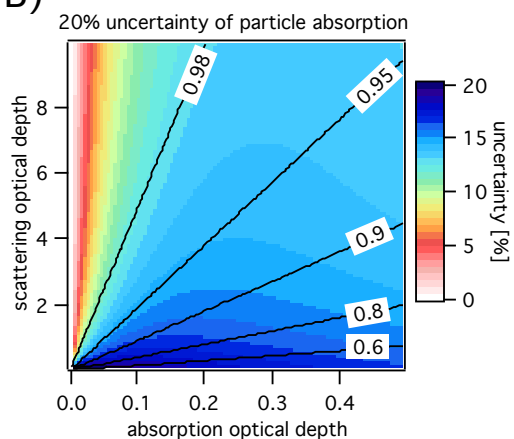

C)

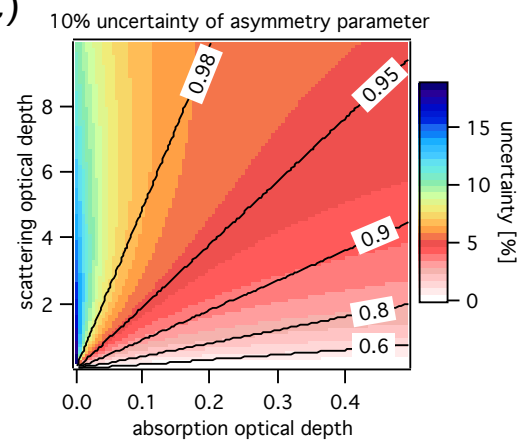

Figure 12. Relative uncertainties in the relative optical depth caused by uncertainties in the particle characterizations: (a) particle scattering, (b) particle absorption, and (c) particle asymmetry parameter. The solid lines indicate constant single-scattering albedos.

A)

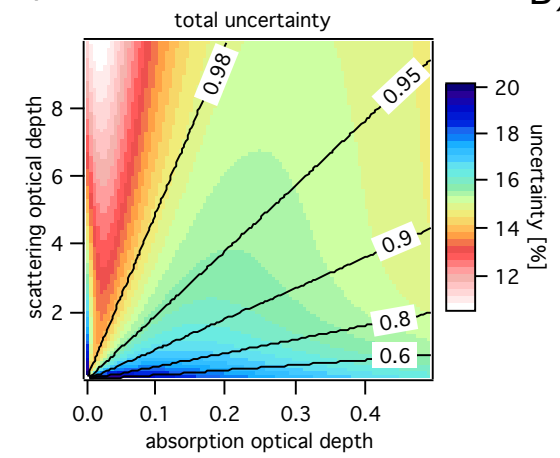

B)

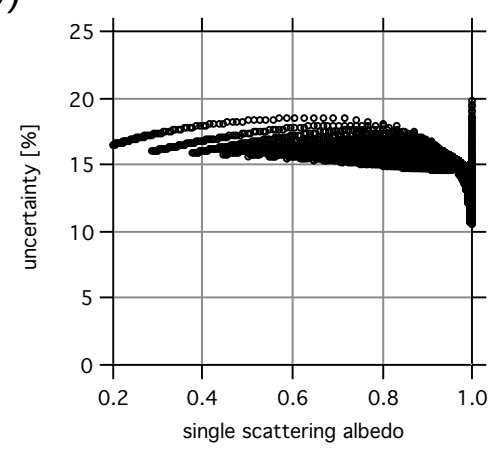

Figure 13. (a) Total uncertainty in the relative optical depth calculated by propagation of uncertainties by model and experimental calibration. (b) Total uncertainties versus particle single-scattering albedo.

plying the CTS method, simultaneous measurements of the particle scattering coefficient and asymmetry parameter are needed, which can be derived from total and backscattering nephelometers. In the present paper the CTS method is introduced for PSAPs. However, the method can be implemented for any other filter-based absorption photometer that measures light transmittance, e.g., the Aethalometer, after measuring the optical properties of the filter and the responses to absorbing and non-absorbing particles.
The CTS method is based on a two-stream radiative transfer model. The model was initialized using measured optical properties of the particle filter and bound to experimentally based calibrations. Calibrations for particles with low singlescattering albedos were taken from the literature, whereas a new calibration for reducing artifacts due to particle scattering was developed. A dependence on the particle asymmetry parameter was found and included in the CTS method. Uncertainties in the model were simulated and discussed and the total uncertainty for retrieving particle absorption coef- 
A) Relative uncertainty of retrieved absorption coefficients using CTS-B1999

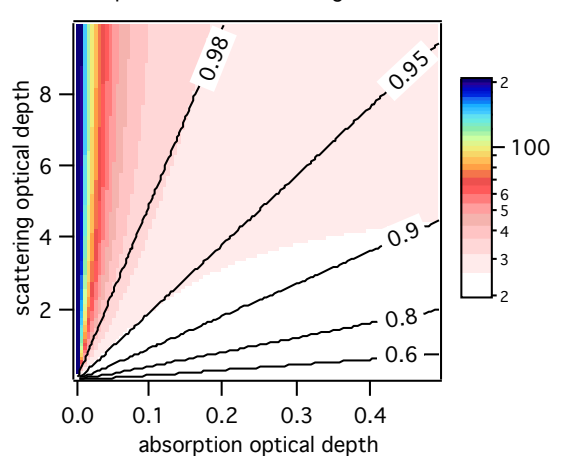

B)
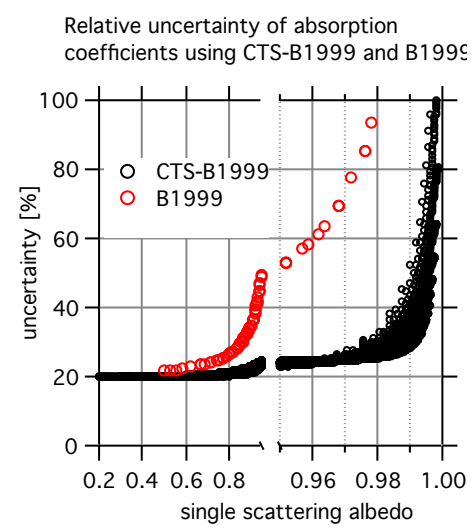

Figure 14. (a) Total relative uncertainty in absorption coefficients retrieved by CTS using the B1999 black aerosol calibration (CTS-B1999). The uncertainty is calculated by error propagation of model and calibration uncertainties. (b) Total uncertainty in absorption coefficients versus particle single-scattering albedo for CTS-B1999 (black) and for B1999 (red).
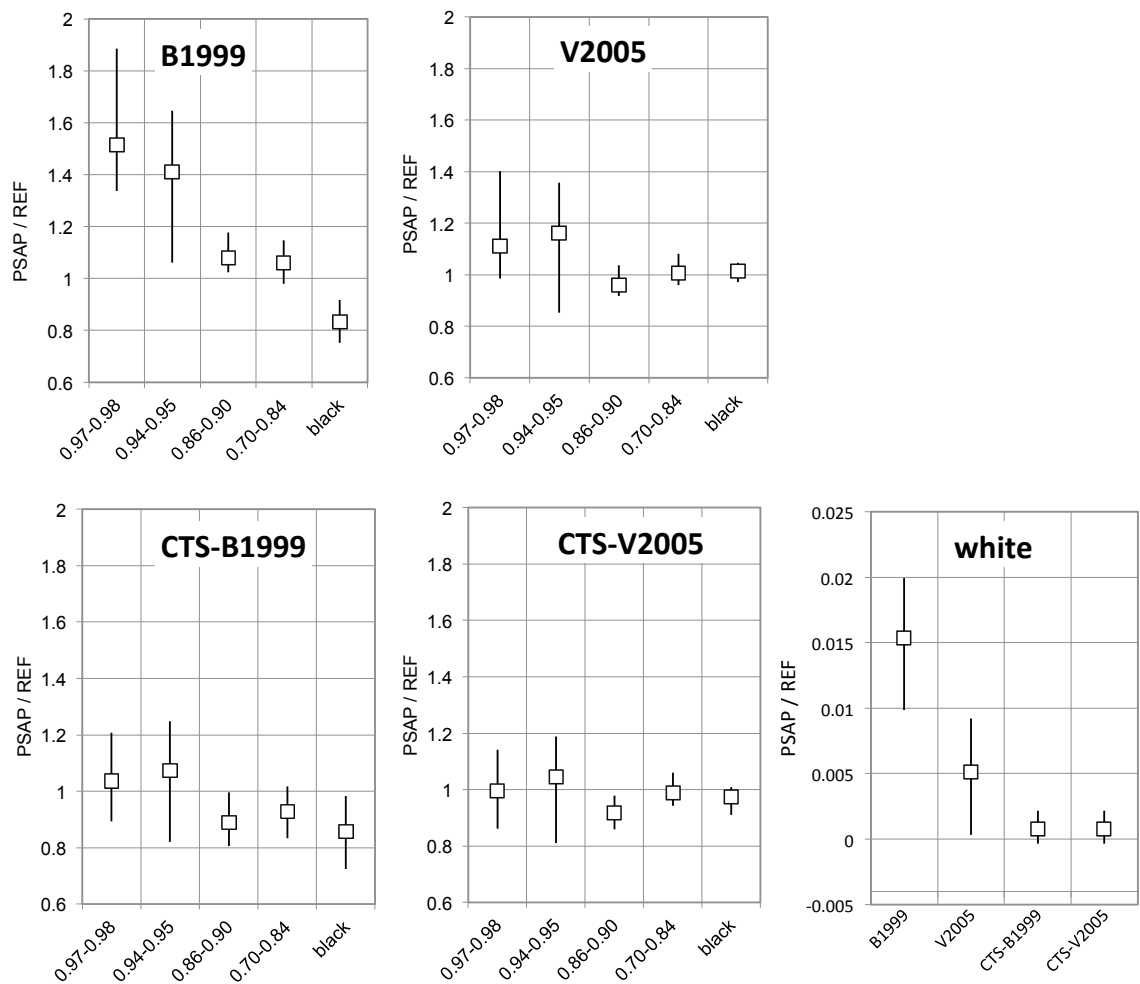

Figure 15. Recalculated PSAP absorption coefficients of the RAOS campaign. The left four plots show absorption coefficients for the two CTS corrections and the B1999 and V2005 corrections divided by the reference absorption. In each subplot, median values (open squares) and 25th and 75th percentiles (whiskers) are shown for ranges of single-scattering albedos. The right plot shows the apparent absorption coefficient measured by the three PSAP correction schemes divided by the scattering coefficient for experiments with white particles.

ficients was derived. The CTS method shows significantly smaller uncertainties for single-scattering albedos larger than 0.9 compared to the well-established correction by Bond et al. (1999). For example, the uncertainties in the B1999 and CTS corrections at a single-scattering albedo of 0.98 are 100 and $30 \%$, respectively. The uncertainties in both methods for single-scattering albedos below 0.8 are very similar with val- ues of about $20 \%$. A comparison of correction methods with data from an independent experiment, the Reno Aerosol Optics Study, confirmed the significant improvements for high single-scattering albedos.

The rather high uncertainties at low single-scattering albedos follow from comparison of results of two calibration experiments that differ by $20 \%$. A further reduction of uncer- 
tainties in filter-based absorption photometers requires better experimental calibrations. Additionally, a size-dependent particle penetration depth, and thus a size-dependent sensitivity, should be included in upcoming correction methods. A coupled model of sampling theory and radiative transfer could be a significant step towards explaining differences between different calibration experiments and reducing uncertainties for filter-based absorption photometers. 


\section{Appendix A: Reformulation of B1999 correction}

The filter transmission function for the B1999 correction without particle scattering is given by

$f_{\mathrm{B} 1999}(\tau(t)):=\frac{\sigma_{\mathrm{ap}}(t)}{\sigma(t)}=\frac{1}{c_{1} \cdot \tau(t)+c_{2}}$.

First, the independent variable $t$ is replaced by the length $l$, which is the column of air sucked through the filter. Using the basic relations $\delta(l)=-\ln (\tau(l))$ and $\sigma(l)=\frac{\mathrm{d}}{\mathrm{d} l} \delta(l)$, we get

$\sigma_{\mathrm{ap}}(l)=\frac{\frac{\mathrm{d}}{\mathrm{d} l} \delta(l)}{c_{1} \cdot e^{-\delta(l)}+c_{2}}$.

Integration of Eq. (A2) in the interval $l=[0, \mathrm{~L}]$ and using the relation $\int_{0}^{L} \sigma_{\text {ap }}(l) \mathrm{d} l=\delta_{\text {ap }}(L)-\delta_{\text {ap }}(0)$ leads to

$\delta_{\text {ap }}(L)-\delta_{\text {ap }}(0)=\int_{0}^{L}\left[\frac{\frac{\mathrm{d}}{\mathrm{d} l} \delta(l)}{c_{1} \cdot e^{-\delta(l)}+c_{2}}\right] \mathrm{d} l$.

The right-hand side may be rewritten as

$\ldots=\int_{0}^{L}\left[\frac{e^{\delta(l)} \frac{\mathrm{d}}{\mathrm{d} l} \delta(l)}{c_{1}+c_{2} e^{\delta(l)}}\right] \mathrm{d} l$.

Substituting $x(l)=c_{1}+c_{2} e^{\delta(l)}$ and $\frac{\mathrm{d}}{\mathrm{d} l} x(l)=c_{2} e^{\delta(l)} \frac{\mathrm{d}}{\mathrm{d} l} \delta(l)$ leads to

$$
\begin{aligned}
\ldots=\int_{0}^{L}\left[\frac{\frac{1}{c_{2}} \frac{\mathrm{d}}{\mathrm{d}} x(l)}{x(l)}\right] \mathrm{d} l & =\frac{1}{c_{2}} \int_{0}^{L}\left[\frac{\mathrm{d}}{\mathrm{d} l} \ln (x(l))\right] \mathrm{d} l \\
& =\frac{\ln (x(L))-\ln (x(0))}{c_{2}} .
\end{aligned}
$$

With boundary conditions for an initially unloaded filter $\delta_{\text {ap }}(0)=0$ and $\delta(0)=0$, Eq. (A3) can be written as

$\delta_{\text {ap }}(L)=\frac{\ln \left(c_{1}+c_{2} e^{\delta(L)}\right)-\ln \left(c_{1}+c_{2}\right)}{c_{2}}$.

Further reformulation to separate the filter optical depth yields

$$
\begin{array}{r}
\ln \left(c_{1}+c_{2} e^{\delta(L)}\right)=c_{2} \delta_{a p}(L)+\ln \left(c_{1}+c_{2}\right), \\
\Leftrightarrow c_{2} e^{\delta(L)}=e^{c_{2} \delta_{a p}(L)+\ln \left(c_{1}+c_{2}\right)}-c_{1}, \\
\Leftrightarrow \delta(L)=\ln \left(\frac{e^{c_{2} \delta_{\mathrm{ap}}(L)+\ln \left(c_{1}+c_{2}\right)}-c_{1}}{c_{2}}\right) \\
=\ln \left(\frac{\left(c_{1}+c_{2}\right) e^{c_{2} \delta_{\mathrm{ap}}(L)}-c_{1}}{c_{2}}\right) .
\end{array}
$$

\section{Appendix B: Reformulation of V2005 correction}

The filter transmission function for non-scattering particles is given by

$f_{\mathrm{V} 2005}(\tau(t)):=\frac{\sigma_{\mathrm{ap}}(t)}{\sigma(t)}=c_{1}+c_{2} h_{0} \ln (\tau(t))$.

The independent variable $t$ is replaced by the length $l$, and with relations $\delta=-\ln (\tau)$ and $\sigma(l)=\frac{\mathrm{d}}{\mathrm{d} l} \delta(l)$ Eq. (B1) may be written as

$c_{1} \frac{\mathrm{d}}{\mathrm{d} l} \delta(l)-c_{2} h_{0} \delta(l) \frac{\mathrm{d}}{\mathrm{d} l} \delta(l)=\sigma_{\mathrm{ap}}(l)$.

Equation (B2) is solved by integration over the interval $l=[0, \mathrm{~L}]$

$\int_{0}^{L}\left(c_{1} \frac{\mathrm{d}}{\mathrm{d} l} \delta(l)-c_{2} h_{0} \delta(l) \frac{\mathrm{d}}{\mathrm{d} l} \delta(l)\right) \mathrm{d} l=\int_{0}^{L} \sigma_{\mathrm{ap}}(l) \mathrm{d} l$.

The solution of the integral equation is

$c_{1} \delta(L)-\frac{1}{2} c_{2} h_{0} \delta(L)^{2}-c_{1} \delta(0)+\frac{1}{2} c_{2} h_{0} \delta(0)^{2}=$

$\delta_{\text {ap }}(L)-\delta_{\text {ap }}(0)$.

Rearranging of Eq. (B4) and using the boundary conditions for an initially unloaded filter $\delta(0)=0$ and $\delta_{\text {ap }}(0)=0$ yields

$c_{1} \delta(L)-\frac{1}{2} c_{2} h_{0} \delta(L)^{2}=\delta_{a p}(L)$.

The solution of the quadratic equation is

$\delta(L)=\sqrt{\left(\frac{c_{1}}{c_{2} h_{0}}\right)^{2}-\frac{2 \delta_{\mathrm{ap}}(L)}{c_{2} h_{0}}}+\frac{c_{1}}{c_{2} h_{0}}$.

The second solution of the quadratic equation does not fulfil the conditions for initially unloaded filters. 


\section{Appendix C}

Table C1. Nomenclature.

\begin{tabular}{|c|c|}
\hline \multicolumn{2}{|c|}{ Abbreviations } \\
\hline PSAP & Particle Soot Absorption Photometer \\
\hline MAAP & Multi-Angle Absorption Photometer \\
\hline B1999 & PSAP correction according to Bond et al. (1999) \\
\hline V2005 & PSAP correction according to Virkkula et al. (2005) \\
\hline CTS & constrained two-stream model \\
\hline CTS-B1999 & CTS correction using B1999 parameterization \\
\hline CTS-V2005 & CTS correction using V2005 parameterization \\
\hline \multicolumn{2}{|c|}{ Commonly used symbols } \\
\hline$A$ & sample spot area \\
\hline$Q$ & volume flow rate \\
\hline$\Delta t$ & time between two intensity readings \\
\hline$I$ & intensity \\
\hline$\sigma$ & attenuation coefficient \\
\hline$\sigma_{\text {ap }}$ & particle absorption coefficient \\
\hline$\sigma_{\mathrm{sp}}$ & particle scattering coefficient \\
\hline$C_{\mathrm{sp}}$ & particle scattering cross section \\
\hline$\tau$ & relative transmittance \\
\hline$\omega_{0}$ & single-scattering albedo \\
\hline$g_{\mathrm{p}}$ & particle asymmetry parameter \\
\hline \multicolumn{2}{|c|}{ Symbols used for CTS } \\
\hline $\mathrm{CTS}(\ldots)$ & CTS model; abbreviation for Eq. (24) \\
\hline$g$ & scattering-weighted asymmetry parameter of particles and fibers \\
\hline$g_{\mathrm{f}}$ & average asymmetry parameter of fibers \\
\hline$f_{\mathrm{B} 1999}$ & filter transmission function according to the B1999 correction \\
\hline$f_{\mathrm{V} 2005}$ & filter transmission function according to the V2005 correction \\
\hline$F_{\mathrm{a}}$ & sensitivity of relative optical depth to absorption optical depth \\
\hline$F_{\mathrm{s}}$ & sensitivity of relative optical depth to scattering optical depth \\
\hline$F_{\mathrm{f}}$ & $\begin{array}{l}\text { mixed-term sensitivity function of relative optical depth to } \\
\text { combined absorption and scattering optical depth }\end{array}$ \\
\hline$R$ & reflectance of a single layer \\
\hline$R_{2 \mathrm{~L}}$ & reflectance of two layers \\
\hline$T$ & transmittance of a single layer \\
\hline$T_{2 \mathrm{~L}}$ & transmittance of two layers \\
\hline$\delta_{\text {tot }}$ & total optical depth of filter \\
\hline$\delta$ & relative optical depth (change in $\delta_{\text {tot }}$ after collecting particle) \\
\hline$\delta_{\mathrm{e}}$ & extinction optical depth (i.e., scattering + absorption) \\
\hline$\delta_{\text {ap }}$ & particle absorption optical depth \\
\hline$\delta_{\mathrm{sp}}$ & particle scattering optical depth \\
\hline$\delta_{\mathrm{af}}$ & absorption optical depth of unloaded filter \\
\hline$\delta_{\mathrm{sf}}$ & scattering optical depth of unloaded filter \\
\hline$\eta_{\mathrm{f}}$ & fractional thickness of particle layer in a two-layer system \\
\hline$\mu_{1}$ & constant accounting for multiple scattering in two-stream models \\
\hline \multicolumn{2}{|c|}{ Parameters used for experimental characterization of optical properties of filters } \\
\hline$S$ & intensities \\
\hline$\alpha_{\text {sphere }}$ & intensity throughput of integrating sphere \\
\hline$c$ & relative intensity loss of integrating sphere \\
\hline \multicolumn{2}{|l|}{ Subscripts } \\
\hline f & filter \\
\hline $\mathrm{p}$ & particle \\
\hline \multicolumn{2}{|l|}{ Superscripts } \\
\hline $\exp$ & experimental results of calibration experiments \\
\hline $\bmod$ & model results \\
\hline meas & measured with absorption photometer \\
\hline
\end{tabular}


Acknowledgements. We gratefully thank all participants of the RAOS experiment for providing us with data.

Edited by: J.-P. Putaud

\section{References}

Anderson, T. L. and Ogren, J. A.: Determining aerosol radiative properties using the TSI 3563 integrating nephelometer, Aerosol Sci. Technol., 29, 57-69, 1998.

Anderson, T. L., Covert, D. S., Marshall, S. F., Laucks, M. L., Charlson, R. J., Waggoner, A. P., Ogren, J. A., Caldow, R., Holm, R. L., Quant, F. R., Sem, G. J., Wiedensohler, A., Ahlquist, N. A., and Bates, T. S.: Performance characteristics of a high-sensitivity, three-wavelength, total scatter/backscatter nephelometer, J. Atmos. Ocean. Tech., 13, 967-986, 1996.

Andreae, M. O., Schmid, O., Yang, H., Chand, D., Yu, J. Z., Zeng, L. M., and Zhang, Y. H.: Optical properties and chemical composition of the atmospheric aerosol in urban Guangzhou, China, Atmos. Environ., 42, 6335-6350, 2008.

Andrews, E., Sheridan, P. J., Fiebig, M., McComiskey, A., Ogren, J. A., Arnott, P., Covert, D., Elleman, R., Gasparini, R., Collins, D., Jonsson, H., Schmid, B., and Wang, J.: Comparison of methods for deriving aerosol asymmetry parameter, J. Geophys. Res.Atmos., 111, D05S04, doi:10.1029/2004JD005734, 2006.

Arnott, W. P., Hamasha, K., Moosmüller, H., Sheridan, P. J., and Ogren, J. A.: Towards aerosol light-absorption measurements with a 7-wavelength Aethalometer: Evaluation with a photoacoustic instrument and 3-wavelength nephelometer, Aerosol Sci. Tech., 39, 17-29, 2005.

Baumgardner, D., Popovicheva, O., Allan, J., Bernardoni, V., Cao, J., Cavalli, F., Cozic, J., Diapouli, E., Eleftheriadis, K., Genberg, P. J., Gonzalez, C., Gysel, M., John, A., Kirchstetter, T. W., Kuhlbusch, T. A. J., Laborde, M., Lack, D., Müller, T., Niessner, R., Petzold, A., Piazzalunga, A., Putaud, J. P., Schwarz, J., Sheridan, P., Subramanian, R., Swietlicki, E., Valli, G., Vecchi, R., and Viana, M.: Soot reference materials for instrument calibration and intercomparisons: a workshop summary with recommendations, Atmos. Meas. Tech., 5, 1869-1887, doi:10.5194/amt-51869-2012, 2012.

Bohren, C. F.: Multiple-scattering of light and some of its observable consequences, Am. J. Phys., 55, 524-533, 1987.

Bohren, C. F. and Huffman D. R.: Absorption and Scattering of Light by Small Particles, John Wiley \& Sons, Inc, New York, 544 pp., 1983.

Bond, T. C., Anderson, T. L., and Campbell, D.: Calibration and intercomparison of filter-based measurements of visible light absorption by aerosols, Aerosol Sci. Technol., 30, 582-600, 1999.

Bond, T. C., Covert, D. S., and Müller, T.: Truncation and AngularScattering Corrections for Absorbing Aerosol in the TSI 3563 Nephelometer, Aerosol Sci. Technol., 43, 866-871, 2009.

Chandrasekhar, S.: Radiative Transfer, Oxford University Press, London, 1950.

Clarke, A. D.: Integrating Sandwich: A New Method of Measurement of the Light Absorption Coefficient for Atmospheric Particles, Appl. Optics, 21, 3011-3020, 1982.

Collaud Coen, M., Weingartner, E., Apituley, A., Ceburnis, D., Fierz-Schmidhauser, R., Flentje, H., Henzing, J. S., Jennings, S.
G., Moerman, M., Petzold, A., Schmid, O., and Baltensperger, U.: Minimizing light absorption measurement artifacts of the Aethalometer: evaluation of five correction algorithms, Atmos. Meas. Tech., 3, 457-474, doi:10.5194/amt-3-457-2010, 2010.

Delene, D. J. and Ogren, J. A.: Variability of aerosol optical properties at four North American surface monitoring sites, J. Atmos. Sci., 59, 1135-1150, 2002.

Fiebig, M. and Ogren, J. A.: Retrieval and climatology of the aerosol asymmetry parameter in the NOAA aerosol monitoring network, J. Geophys. Res.-Atmos., 111, D21204, doi:10.1029/2005jd006545, 2006.

Gorbunov, B., Hamilton, R., and Hitzenberger, R.: Modeling radiative transfer by aerosol particles on a filter, Aerosol Sci. Technol., 36, 123-135, 2002.

Hansen, A. D. A., Rosen, H., and Novakov, T.: The aethalometer - An instrument for the real-time measurement of opticalabsorption by aerosol-particles, Sci. Total Environ., 36, 191-196, 1984.

Heintzenberg, J., Wiedensohler, A., Tuch, T. M., Covert, D. S., Sheridan, P., Ogren, J. A., Gras, J., Nessler, R., Kleefeld, C., Kalivitis, N., Aaltonen, V., Wilhelm, R. T., and Havlicek, M.: Intercomparisons and aerosol calibrations of 12 commercial integrating nephelometers of three manufacturers, J. Atmos. Ocean. Tech., 23, 902-914, 2006.

Hyvärinen, A.-P., Vakkari, V., Laakso, L., Hooda, R. K., Sharma, V. P., Panwar, T. S., Beukes, J. P., van Zyl, P. G., Josipovic, M., Garland, R. M., Andreae, M. O., Pöschl, U., and Petzold, A.: Correction for a measurement artifact of the Multi-Angle Absorption Photometer (MAAP) at high black carbon mass concentration levels, Atmos. Meas. Tech., 6, 81-90, doi:10.5194/amt-681-2013, 2013.

Lee, K. W. and Mukund, R.: Chapter "Filter collection" in Baron, P. A. and Willeke, K., Aerosol Measurement, 2nd Edn., J. Wiley and Sons, 170-178, 2001.

Lin, C., Baker, M., and Charlson, R. J.: Absorption-coefficient of atmospheric aerosol - a method for measurement, Appl. Optics, 12, 1356-1363, 1973.

Liou, K. N.: An Introduction to Atmospheric Radiation, Academic Press, San Diego, CA, 2002.

Lyzenga, D. R.: Note on the Modified Two-Stream Approximation of Sagan and Pollack, Icarus, 19, 240-243, 1973.

Miyazaki, Y., Kondo, Y., Sahu, L. K., Imaru, J., Fukushima, N., and Kano, M.: Performance of a newly designed continuous soot monitoring system (COSMOS), J. Environ. Monitor., 10, 1195-1201, 2008.

Moosmüller, H., Chakrabarty, R. K., and Arnott, W. P.: Aerosol light absorption and its measurement: A review, J. Quant. Spectrosc. Ra., 110, 844-878, 2009.

Moteki, N., Kondo, Y., Nakayama, T., Kita, K., Sahu, L. K., Ishigai, T., Kinase, T., and Matsumi, Y.: Radiative transfer modeling of filter-based measurements of light absorption by particles: Importance of particle size dependent penetration depth, J. Aerosol Sci., 41, 401-412, 2010.

Müller, T., Henzing, J. S., de Leeuw, G., Wiedensohler, A., Alastuey, A., Angelov, H., Bizjak, M., Collaud Coen, M., Engström, J. E., Gruening, C., Hillamo, R., Hoffer, A., Imre, K., Ivanow, P., Jennings, G., Sun, J. Y., Kalivitis, N., Karlsson, H., Komppula, M., Laj, P., Li, S.-M., Lunder, C., Marinoni, A., Martins dos Santos, S., Moerman, M., Nowak, A., Ogren, J. A., Pet- 
zold, A., Pichon, J. M., Rodriquez, S., Sharma, S., Sheridan, P. J., Teinilä, K., Tuch, T., Viana, M., Virkkula, A., Weingartner, E., Wilhelm, R., and Wang, Y. Q.: Characterization and intercomparison of aerosol absorption photometers: result of two intercomparison workshops, Atmos. Meas. Tech., 4, 245-268, doi:10.5194/amt-4-245-2011, 2011.

Nakayama, T., Kondo, Y., Moteki, N., Sahu, L. K., Kinase, T., Kita, K., and Matsumi, Y.: Size-dependent correction factors for absorption measurements using filter-based photometers: PSAP and COSMOS, J. Aerosol Sci., 41, 333-343, 2010.

Ogren, J. A.: Comment on "Calibration and Intercomparison of Filter-Based Measurements of Visible Light Absorption by Aerosols", Aerosol Sci. Technol., 44, 589-591, 2010.

Petzold, A. and Schönlinner, M.: Multi-angle absorption photometry-a new method for the measurement of aerosol light absorption and atmospheric black carbon, J. Aerosol Sci., 35, 421-441, 2004.

Petzold, A., Kramer, H., and Schönlinner, M.: Continuous measurement of atmospheric black carbon using a multi-angle absorption photometer, Environ. Sci. Pollut. R., 4, 78-82, 2002.

Petzold, A., Schloesser, H., Sheridan, P. J., Arnott, W. P., Ogren, J. A., and Virkkula, A.: Evaluation of Multiangle Absorption Photometry for Measuring Aerosol Light Absorption, Aerosol Sci. Technol., 39, 40-51, 2005.
Sagan, C. and Pollack, J. B.: Anisotropic Nonconservative Scattering and the Clouds of Venus, J. Geophys. Res., 72, 469-477, 1967.

Schuster, A.: Radiation through a foggy atmosphere, Astrophys. J., 21, 1-22, 1905.

Sheridan, P. J., Arnott, W. P., Ogren, J. A., Andrews, E., Atkinson, D. B., Covert, D. S., Moosmüller, H., Petzold, A., Schmid, B., Strawa, A. W., Varma, R., and Virkkula, A.: The Reno Aerosol Optics Study: An evaluation of aerosol absorption measurement methods, Aerosol Sci. Technol., 39, 1-16, 2005.

Smith, W.J.: Modern optical engineering (4th ed.), p. 217, McGrawHill, New York, 2008.

Virkkula, A.: Correction of the Calibration of the 3-wavelength Particle Soot Absorption Photometer ( $3 \lambda$ PSAP), Aerosol Sci. Technol., 44, 706-712, 2010.

Virkkula, A., Ahlquist, N. C., Covert, D. S., Arnott, W. P., Sheridan, P. J., Quinn, P. K., and Coffman, D. J.: Modification, calibration and a field test of an instrument for measuring light absorption by particles, Aerosol Sci. Technol., 39, 68-83, 2005.

Weingartner, E., Saathoff, H., Schnaiter, M., Streit, N., Bitnar, B., and Baltensperger, U.: Absorption of light by soot particles: determination of the absorption coefficient by means of aethalometers, J. Aerosol Sci., 34, 1445-1463, 2003. 